\section{备 \\ A view of the current and future role of optical coherence tomography in the management of age-related macular degeneration}

Christian Doppler Laboratory for Ophthalmic Image Analysis, Vienna Reading Center, Medical University of Vienna, Vienna, Austria

Correspondence: U Schmidt-Erfurth, Department of Ophthalmology, Medical University of Vienna, Spitalgasse 23, Vienna 1090, Austria

Tel: +43140400 79310; Fax: +43140400 79320 . E-mail: ursula.schmidterfurth@meduniwien.ac.at

Received: 15 July 2016 Accepted in revised form: 20 September 2016 Published online: 25 November 2016

\begin{abstract}
Optical coherence tomography (OCT) has become an established diagnostic technology in the clinical management of age-related macular degeneration (AMD). OCT is being used for primary diagnosis, evaluation of therapeutic efficacy, and long-term monitoring. Computer-based advances in image analysis provide complementary imaging tools such as OCT angiography, further novel automated analysis methods as well as feature detection and prediction of prognosis in disease and therapy by machine learning. In early AMD, pathognomonic features such as drusen, pseudodrusen, and abnormalities of the retinal pigment epithelium (RPE) can be imaged in a qualitative and quantitative way to identify early signs of disease activity and define the risk of progression. In advanced AMD, disease activity can be monitored clearly by qualitative and quantified analyses of fluid pooling, such as intraretinal cystoid fluid, subretinal fluid, and pigment epithelial detachment (PED). Moreover, machine learning methods detect a large spectrum of new biomarkers. Evaluation of treatment efficacy and definition of optimal therapeutic regimens are an important aim in managing neovascular AMD. In atrophic AMD hallmarked by geographic atrophy (GA), advanced spectral domain (SD)-OCT imaging largely replaces conventional fundus autofluorescence (FAF) as it adds insight into the condition of the neurosensory layers and associated alterations at the level of the RPE and choroid. Exploration of imaging features by computerized methods has just begun but has already opened relevant and reliable horizons for the optimal use of OCT imaging for individualized and population-based
\end{abstract}

U Schmidt-Erfurth, S Klimscha, SM Waldstein and $\mathrm{H}$ Bogunović

management of AMD-the leading retinal epidemic of modern times.

Eye (2017) 31, 26-44; doi:10.1038/eye.2016.227; published online 25 November 2016

Method of literature research

We conducted a comprehensive literature review to provide a clinically relevant overview of current knowledge of features of age-related macular degeneration $(\mathrm{AMD})$ seen on optical coherence tomography (OCT) and their relevance for disease progression. We performed a thorough search of peer-reviewed articles published up to June 2016 on the PubMed database and included relevant papers according to our knowledge of the field. In our review, we describe the pathognomonic value and pathophysiological relevance of features of early/intermediate, neovascular, and advanced atrophic AMD. We summarize data on disease progression in AMD, emphasize pertinent factors for practical patient management, and conclude each section with a perspective of future developments.

Early and intermediate AMD

Drusen, reticular pseudodrusen, and hyperreflective foci are the key pathognomonic features in early disease stages of AMD. They represent dynamic structures evolving over time, with changes often entailing a risk of disease progression. Currently available spectral domain-OCT (SD-OCT) systems provide highresolution, cross-sectional, and volumetric 
visualization of the retina, thereby enabling the measurement and monitoring of numerous characteristic features.

\section{Features of pathognomonic value}

Drusen are one of the earliest signs of AMD and are key to classifying early stages of the disease. For classification, qualitative and quantitative features of the morphology and development of drusen have commonly been assessed by biomicroscopy and color fundus photography. ${ }^{1}$ SD-OCT has strongly enhanced our ability to assess these early features. Its high-resolution, crosssectional imaging properties enable detailed visualization of drusen diameter, height, area, volume, shape, internal reflectivity, and homogeneity as well as a distinct evaluation of the condition of the retinal pigment epithelium (RPE). Examples of characteristic drusen microstructures can be seen in Figure 1. Using delineation of the RPE and the RPE-drusen complex, including reticular pseudodrusen, presenting on OCT as subretinal drusenoid deposits, it is possible to automatically distinguish normal eyes from eyes with intermediate AMD by identifying total retinal volumes and RPEdrusen complex volumes together with RPE-drusen complex abnormal thickening and thinning. ${ }^{2}$

Soft and hard drusen present as RPE elevations on SD-OCT, differing in size and shape with medium to highly reflective sub-RPE material. Their appearance on OCT has been characterized in detail in numerous reports. ${ }^{3-5}$ Convex, homogenous drusen with medium internal reflectivity and without overlying hyperreflective foci account for the most common type of drusen, representing $47 \%$ of drusen (Figure 1c). Around 50\% of soft-indistinct drusen, classified by color fundus photography, show these characteristics. The other 50\%
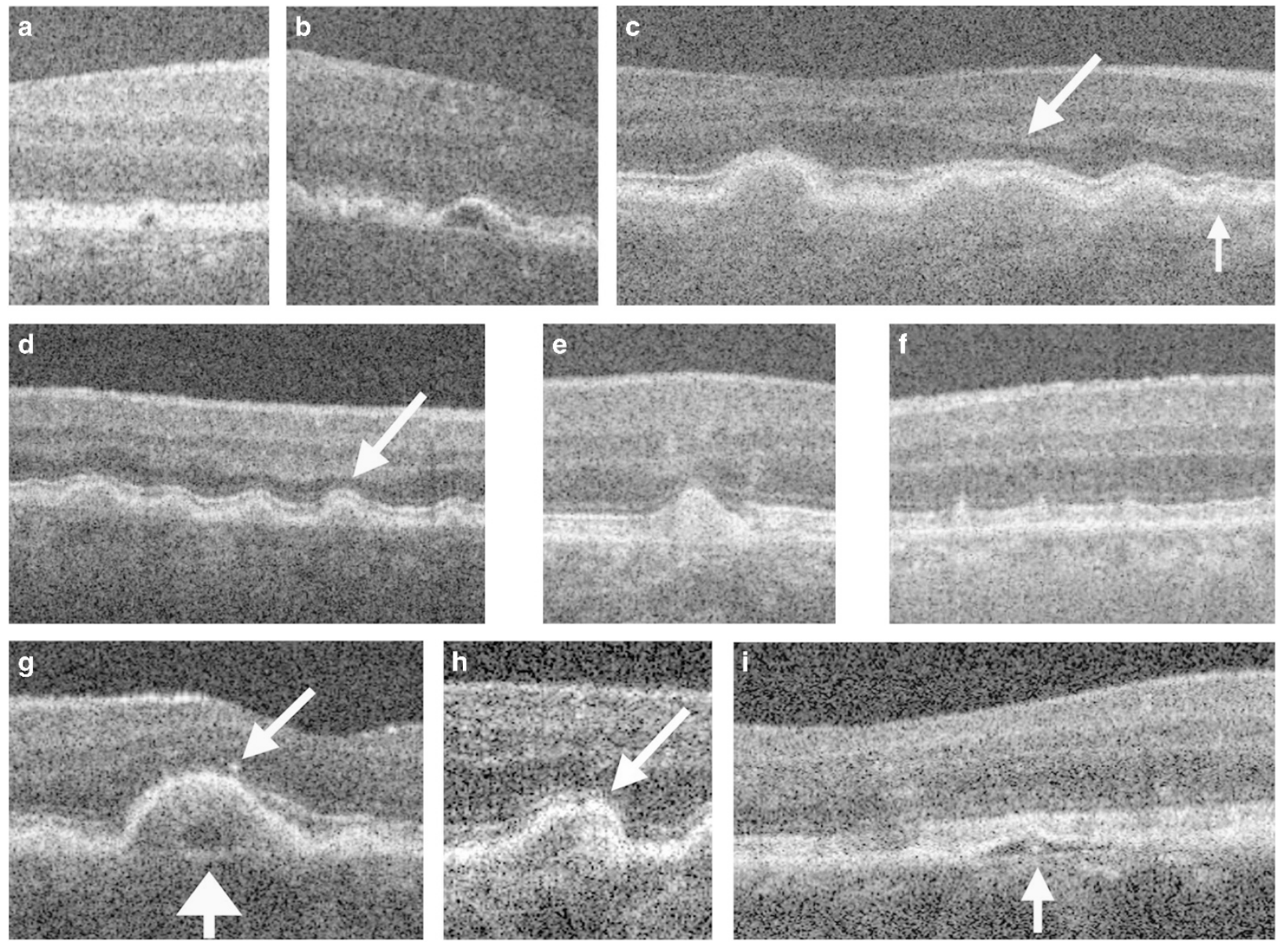

Figure 1 Examples of drusen and retinal morphology in eyes with intermediate AMD, visualized by SD-OCT. (a, b) Drusen with low internal reflectivity. (c, d) Drusen with medium internal reflectivity. Arrows indicate photoreceptor layer thinning overlying such drusen. (e) Druse with high internal reflectivity. (f) Subretinal drusenoid deposit (stage 2). (g) Low-reflective core (wide arrow) within a medium-reflective druse and overlying hyper-reflective focus (narrow arrow). (h) Hyper-reflective core within a medium-reflective druse. (i) Hyper-reflective core within druse with low reflectivity. (Figure from Leuschen $e t a l^{4}$ ). 
show a range of different morphological characteristics on SD-OCT. ${ }^{3}$ Saw-tooth-shaped RPE elevations, which have been associated with hard drusen, ${ }^{5}$ do not show overlying hyperreflective foci, ${ }^{3}$ calcified or refractile drusen usually present as convex structures with hyperreflective dots superimposed on a hyporeflective, non-homogenous background, and occasional shadowing into deeper structures (Figure 1b). Refractile drusen are more likely than soft-indistinct drusen to have overlying hyperreflective foci. ${ }^{5,6}$ Different degrees of RPE integrity and alteration can be distinguished above refractile drusen, dependent on the topographic location of drusen in the posterior pole. Drusen located closer to the central macula mostly lack overlying intact RPE or a continuous $\mathrm{RPE}$ across the base of the druse. A retrospective evaluation of progression found that zones of RPE atrophy, characterized in color fundus photography and fundus autofluorescence (FAF) imaging, spread to the outer regions concentrically over time, suggesting that refractile drusen derive from conventional drusen. ${ }^{6}$

Following drusen progression has become considerably more precise with OCT imaging. Various studies have shown drusen to be dynamic structures running through repeated cycles of volume increase and decrease, with growth more likely than resolution (Figure 2). ${ }^{7,8}$ Drusen seem to grow in a cubic pattern with the amount of drusen accumulation increasing over time, whereas their regression occurs very fast. ${ }^{7}$ A prospective longitudinal natural history study by Yehoshua et $\mathrm{al}^{8}$ suggested larger drusen to show a tendency to decrease in volume.

Certain drusen characteristics identifiable on OCT, such as moderate internal drusen reflectivity with or without sub-RPE hyper-reflectivity, may in some cases be a sign of progressive type 1 choroidal neovascularization (CNV), mimicking drusenoid RPE elevation. ${ }^{9}$ Besides drusen, asymptomatic type $1 \mathrm{CNV}$ can also present within irregularly slightly elevated pigment epithelial detachment (PED). ${ }^{10}$ These lesions, termed vascularized drusen or quiescent $\mathrm{CNV}$, have so far mainly been detected by indocyanine green angiography (ICGA). Recently, they have been successfully characterized using OCT angiography, which relies on blood flow detection in scans obtained successively at the same location. ${ }^{11}$ Neither the natural history of these lesions nor their potentially unfolding implications for disease progression have been thoroughly studied.

Pseudodrusen, or subretinal drusenoid deposits, are recognized as well-defined round or triangular hyperreflective material located above the RPE (Figure 1f). ${ }^{12}$ In a large cross-sectional study of elderly patients, the prevalence of subretinal drusenoid deposits was found to be $32 \%$ overall, $23 \%$ in those without AMD, and $52 \%$ in those with AMD. Among patients with AMD, the prevalence was $49 \%$ in those with early and $79 \%$ in those with intermediate AMD. After adjustment for age, patients presenting with subretinal drusenoid deposits were 3.4 times more likely to have AMD. The prevalence of pseudodrusen was therefore strongly associated with the presence and severity of AMD and increased with age. ${ }^{13}$ The consensus seems to be that subretinal drusenoid deposits are most reliably identified using two en-face imaging modalities: preferably SD-OCT and infrared reflectance imaging. ${ }^{14}$ Recently, however, Schaal et $a l^{15}$ proposed wide-field en-face slab imaging with SD-OCT and swept-source OCT as a new imaging standard in detecting subretinal drusenoid deposits with the potential to replace multimodal imaging. Similar to drusen, subretinal drusenoid deposits have been shown to be dynamic structures, changing in appearance, stage and volume. They have been suggested to develop independently from drusen. ${ }^{16}$ Stage 1 is defined as the diffuse deposition of granular hyperreflective material between the RPE and the retinal ellipsoid zone (EZ) — former inner segment/outer segment (IS/OS) junction. Stage 2 presents with mounds of accumulated material sufficient to alter the contour of the EZ boundary. Stage 3 consists of thicker material breaking through the EZ. ${ }^{12}$ A fourth stage, defined by Querques et $a l^{17}$ presents as fading of subretinal deposits owing to reabsorption and migration within the inner retinal layers.

Pigmentary changes in color fundus photography have been associated with disease progression. ${ }^{1}$ In SD-OCT, they present as hyperreflective foci, discrete, well-defined lesions with high reflectivity within the neurosensory retina. ${ }^{18}$ In a large prospective cross-sectional study of OCT features in intermediate AMD, hyperreflective foci were shown to be present in $>50 \%$ of eyes with AMD and in none of the healthy control eyes. Drusen with a central core, with low or high internal reflectivity, are more likely to have overlying hyperreflective foci. ${ }^{4}$ A 2-year prospective analysis of 300 eyes presenting with intermediate AMD showed hyperreflective foci to progressively proliferate and migrate toward the inner retinal layers (Figure 3). ${ }^{19}$ Prominent hyperreflective haze in the outer nuclear layer (ONL) above drusen has been described as an additional manifestation of a hyperreflective abnormality and is associated with drusen in $67 \%$ of foveal SD-OCT scans of eyes with nonneovascular AMD, compared with $18 \%$ of control eyes showing only faint haze. ${ }^{20}$

\section{Risk of progression toward advanced AMD}

The known increased risk of AMD progression for patients presenting with medium ( $\geq 63 \mu \mathrm{m})$ or especially large $(\geq 125 \mu \mathrm{m})$ drusen on color fundus photography ${ }^{1}$ has spurred on researchers to identify drusen-related features 

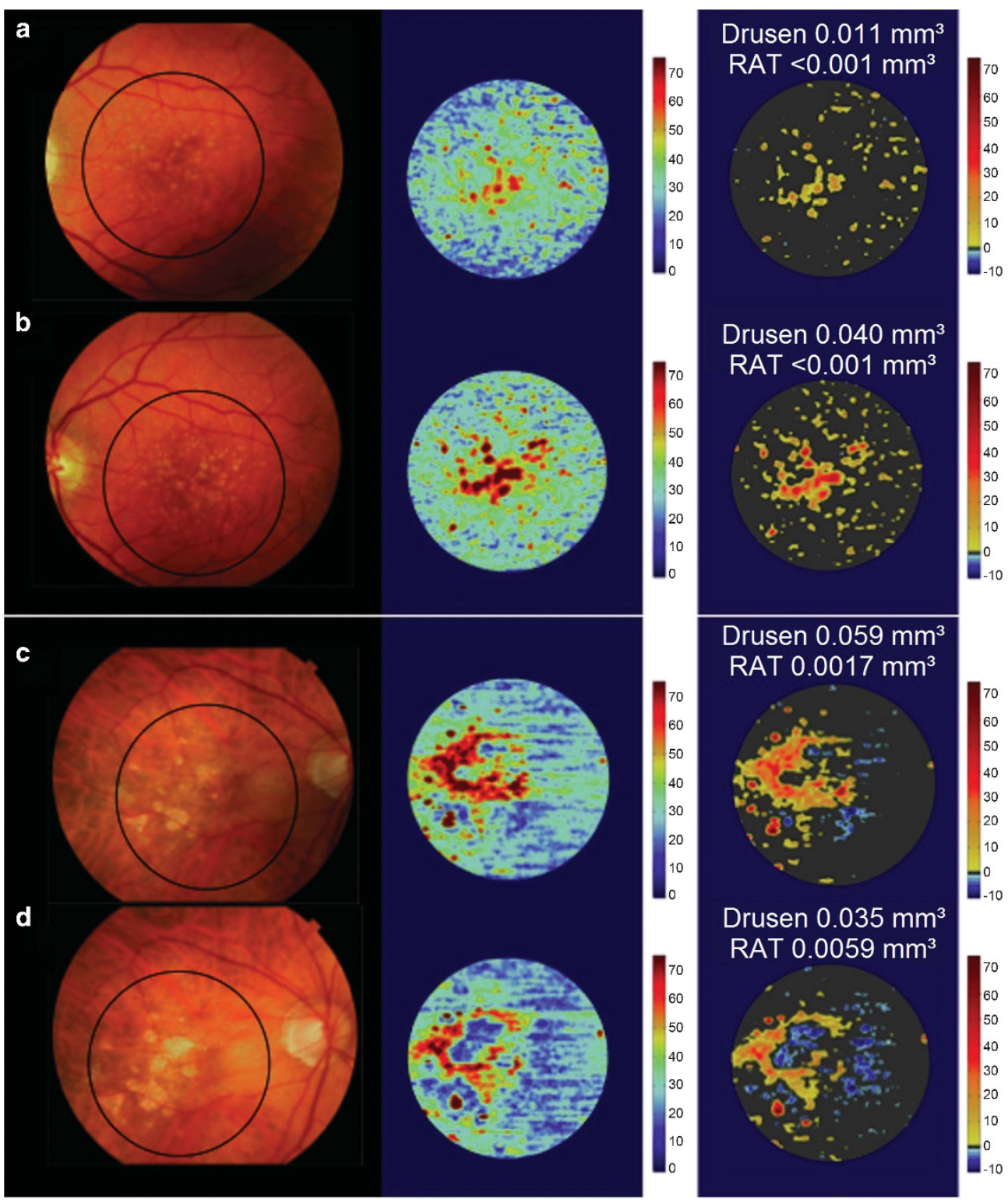

Figure 2 Longitudinal change in drusen volume on SD-OCT maps. Color fundus photographs (left column), SD-OCT thickness maps of RPE-drusen complex (middle column), and maps combining RPE-drusen complex abnormal thickening (drusen) in orange tones and RPE-drusen complex abnormal thinning in blue tones. Eye with AMD presenting drusen volume increase from baseline (a) to year 2 (b). AMD eye with partial resolution of drusen volume from baseline (c) to year 2 (d). RAT = RPE - complex abnormal thinning (Figure modified from Folgar et $a l^{18}$ ).

detectable on OCT preceding the progression to late AMD. The microstructure of drusen has been studied in detail. ${ }^{3}$ Internal drusen cores and heterogeneous reflectivity as well as a change in reflectivity over time from homogeneous to heterogeneous patterns have been associated with the onset of focal RPE atrophy, defined as loss of RPE and EZ bands and resulting in increased signal transmission below the Bruch's membrane (BM) accompanied by loss of the external limiting membrane (ELM) and ONL. Furthermore, greater height of drusenoid lesions, including drusenoid PED, increases the risk of focal atrophy. ${ }^{4,21} \mathrm{~A}$ focal loss of the overlying photoreceptor layer has also been shown to be associated with drusen, especially drusen height. ${ }^{20}$

In line with previous studies based on color fundus photography, several investigators have shown that eyes with higher baseline drusen volume have an increased risk of progression to either $\mathrm{CNV}$ or geographic atrophy (GA). ${ }^{8,18,22}$ In a 2-year observational study, Folgar et al ${ }^{18}$ showed an odds ratio (OR) of 1.31 for developing CNV 

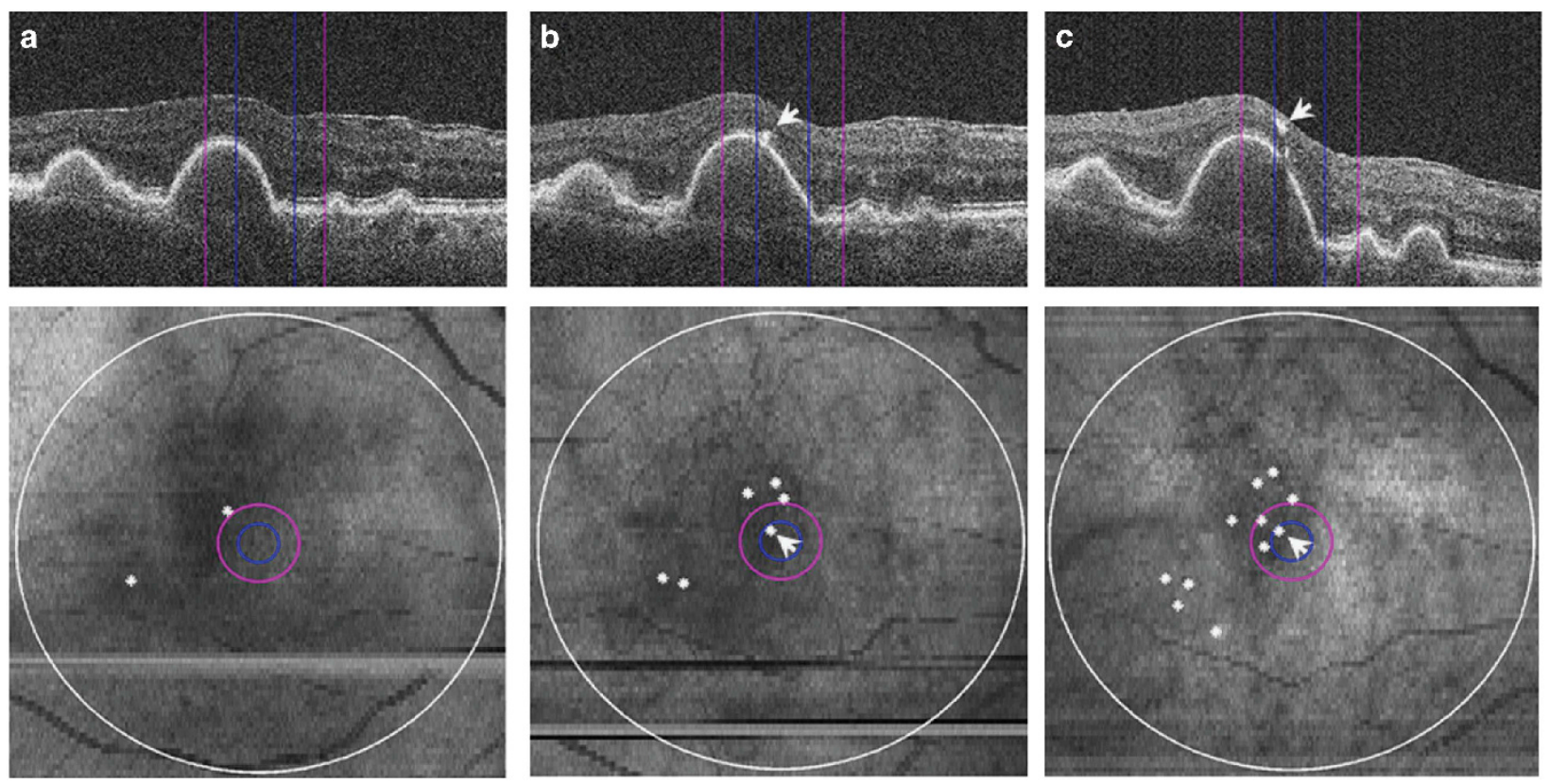

Figure 3 SD-OCT visualization of hyper-reflective foci proliferation and migration toward inner retinal layers. Magnified SD-OCT images (top) and corresponding SD-OCT-derived retinal maps (bottom). 500- $\mu \mathrm{m}, 1-\mathrm{mm}$, and 6-mm fields are indicated by blue, purple, and white circles, respectively. (a) baseline; (b) 1-year follow-up; (c) 2-year follow-up. Progressive migration of hyper-reflective foci (arrowheads) and their proliferation (white dots) can be observed. (Figure from Christenbury $e t a l^{19}$ ).

for every $0.1 \mathrm{~mm}^{3}$ increase in RPE-drusen complex abnormal thickening (representing drusen) in 266 eyes. Complementary, abnormal thinning of the RPE-drusen complex likewise increased significantly over 2 years (Figures $2 \mathrm{c}$ and $\mathrm{d}$ ). A higher thinning volume at baseline was associated with progression to central GA in eyes with baseline non-central GA. In their natural history study, Yehoshua $\mathrm{et}^{\mathrm{al}} \mathrm{l}^{8}$ identified eyes with a large initial drusen volume as more likely to present with a decrease in total drusen volume and area within 2 years. In a subgroup of $15 \%$ of eyes where the baseline drusen volume decreased $\geq 50 \%$ on a cube root scale (equal to $88 \%$ of baseline drusen volume expressed as $\mathrm{mm}^{3}$ ), almost $90 \%$ of eyes progressed to late AMD, $64 \%$ to GA, and $23 \%$ to neovascular AMD.

As eyes presenting with drusen regression tend to progress to advanced AMD, and in return, eyes rarely progress without foregoing drusen regression, this rapidly occurring event seems to present a key factor in conversion to advanced AMD. 7,8 Marked by RPE loss, refractile drusen appear to represent a stage of drusen regression preceding the development of GA. ${ }^{6}$ The high axial resolution of SD-OCT offers ideal properties to investigate subtle changes preceding drusen regression. Precursors of drusen-associated atrophy, named 'nascent geographic atrophy', consist of a subsidence of the outer plexiform layer (OPL) and inner nuclear layer as well as the development of a hyporeflective wedge-shaped band within the boundaries of the OPL. These unique features are often accompanied by a disruption of the adjacent RPE and ELM, the presence and migration of hyperreflective foci, and increased transmission below the RPE. An average of a year has been described to lie between the occurrence of nascent GA and drusenassociated GA. Risk factors for the development of nascent GA were found to be hyperreflective foci in the affected eye and nascent GA in the fellow eye. ${ }^{23}$ The presence of hyperreflective foci is also directly associated with the onset of RPE atrophy. ${ }^{4,19,21}$ Migration and new onset of hyperreflective foci correlates with the risk of progression to focal atrophy. ${ }^{4,21}$

In a large prospective study that included 799 eyes of 455 participants in normal macular health of the Age Related Eye Disease Study (AREDS), eyes presenting with subretinal drusenoid deposits were 2.24 times more likely to develop AMD during a 3-year follow-up. ${ }^{24}$

Furthermore, an increased relative risk of developing late AMD when presenting with subretinal drusenoid deposits has been reported in several studies; however, none of them used SD-OCT in the diagnosis of pseudodrusen. ${ }^{14}$ Observable by OCT, subretinal drusenoid deposits undergo a characteristic lifecycle of growth, invasion into the adjacent $\mathrm{EZ}$, and finally regression. In a retrospective study, Spaide ${ }^{25}$ found regression of subretinal drusenoid deposits to correlate with outer retinal atrophy and a decrease in choroidal 
thickness, often located in the superior macular area. The regression of subretinal drusenoid deposits has up until now not been associated with progression of intermediate-to-late AMD.

\section{Patient management in early and intermediate AMD}

OCT presents an ideal technology for the diagnosis and assessment of disease progression of early and intermediate stages of AMD. Drusen, as the predominant pathognomonic feature, can be monitored in detail based on their characteristic microstructure. High-risk drusen, indicating the development of local RPE atrophy and, therefore, requiring intensified monitoring, often present with heterogeneous internal reflectivity. A change from homogeneous to heterogeneous internal reflectivity, a drusen core, and overlying hyperreflective foci can further precede this condition. ${ }^{4,21}$ Even closer monitoring is indicated following drusen regression, as it marks an event often preceding progression to both dry and exudative forms of late AMD. Volumetric OCT drusen measurements are a reliable tool for quantifying drusen regression, as drusen volume measured by SD-OCT has been shown to decrease by $>50 \%$ of the cube root volume without any noticeable correlate in color fundus photography. 7,8 These measurements give insight into drusen growth and regression as well as RPE thinning, an important predictor for the development of central GA. ${ }^{18}$ Changes preceding drusen regression present markers allowing clinicians to adjust follow-up intervals and potentially detect signs of late AMD before severe and irreversible vision loss occurs. Both refractile drusen and 'nascent GA' present such changes identifiable on OCT. These changes are not only a signal for intensifying monitoring but should also be used for designing clinical end points in future studies. ${ }^{6,23,26}$ The need for identification of asymptomatic CNV lesions within drusen and drusenoid PEDs has paved the way for the increasing importance of OCT angiography in the clinical assessment of early stages of the disease. ${ }^{11}$ With SD-OCT offering the highest sensitivity for detecting

pseudodrusen ${ }^{14}$ and wide-field en-face swept-source OCT and SD-OCT slab imaging possibly replacing multimodal imaging thereof, ${ }^{15}$ OCT is the ideal method for identifying these lesions. Systematic scanning for subretinal drusenoid deposits is recommendable as their presence poses a risk factor for early onset of AMD and rapid progression to late AMD, particularly in combination with soft drusen. ${ }^{14}$ Pseudodrusen regression has been proposed to present a form of advanced AMD, different from neovascular and atrophic late AMD. Further studies are needed to investigate the progression of AMD and subretinal drusenoid deposits in parallel and clearly characterize the correlation of lesion stages with different forms of late AMD. ${ }^{25}$ Naturally, the reliability of the evaluation of

SD-OCT volumes is dependent on the interpreter's experience and may be poorly reproducible. However, Khanifar and co-workers ${ }^{3}$ reported overall good interobserver agreement on drusen microstructure: highest for reflectivity and shape followed by the presence of hyperreflective foci and internal homogeneity.

Hyperpigmentation and larger drusen have been reported to be detected to a greater extent on SD-OCT and smaller drusen to have a trend for greater detection in color fundus photography. ${ }^{27}$

\section{Future technology and perspectives}

Computer-based analysis of features offers solutions for many issues introduced by the subjective assessment of huge amounts of imaging data, which have made meaningful evaluation unfeasible in a clinical setting. Features of early AMD have proven to be exceptionally suitable for computational image analysis. Besides several research algorithms, a Food and Drug Administration (FDA)-approved software ('Advanced RPE Analysis tool'), which measures drusen volume and RPE atrophy, has been integrated into the Cirrus OCT device (Carl Zeiss Meditec, Dublin, CA, USA) and has thereby become commonly available. The algorithm uses segmentation of the RPE and a virtual RPE floor free of deformations to generate thickness maps of sub-RPE drusen $^{28}$ and determines RPE atrophy by identifying RPE loss combined with hyper-reflectivity in the choroid. Several papers have reported successful applications of the software. ${ }^{8,22,29}$ A framework for drusen segmentation, based on graph theory and dynamic programming, designed by Chiu and co-workers, was applied in the AREDS2 Ancillary SD-OCT Study. It provides measurements of RPE-drusen complex volumes, including subretinal drusenoid deposits, and has been proven effective for assessing disease progression. $2,18,30$ Advances in computational image analysis have further created possibilities of individual risk assessment for disease progression. De Sisternes et $a l^{31}$ used a fully automated pipeline for segmentation and feature characterization in a statistical model designed to estimate the likelihood of conversion from early and intermediate to exudative AMD and achieved an accuracy of the predictive model of a mean area under the receiver operating characteristic curve of 0.74 . In terms of imaging hardware, polarization-sensitive OCT presents an advanced technique, extending the concept of OCT. It uses intrinsic, tissue-specific information derived from light-tissue interactions that cause changes in the polarization state of the backscattered light. Its ability to selectively segment the RPE layer based on its 
depolarization characteristics is of particular interest in image analysis of early and intermediate AMD. Combined with a virtual drusen-free RPE floor, polarization-sensitive OCT provides reliable drusen segmentation as well as additional polarization-derived morphological characteristics of drusen. ${ }^{7,32}$ Figure 4 shows visualization of these characteristics by PS-OCT.

\section{Neovascular AMD with CNV}

Neovascular or exudative AMD is characterized by the formation of abnormal choroidal vessels breaking through the BM and proliferating into the sub-RPE space. These vessels termed CNV can further invade beyond the RPE into the subretinal and intraretinal space. Exudation due to the immaturity of vessels often results in fluid accumulation in different compartments depending on the level of CNV invasion. ${ }^{33}$ Treatment with intravitreal injections of antivascular endothelial growth factor (anti-VEGF) has become state-of-the-art therapy for exudative advanced disease, allowing substantial morphological recovery and long-term stabilization of visual function in most patients. ${ }^{34}$ Identification of pathognomonic features using OCT has generated a more profound understanding of the pathophysiology and response patterns of disease during anti-VEGF therapy, with the future aim of defining optimal treatment

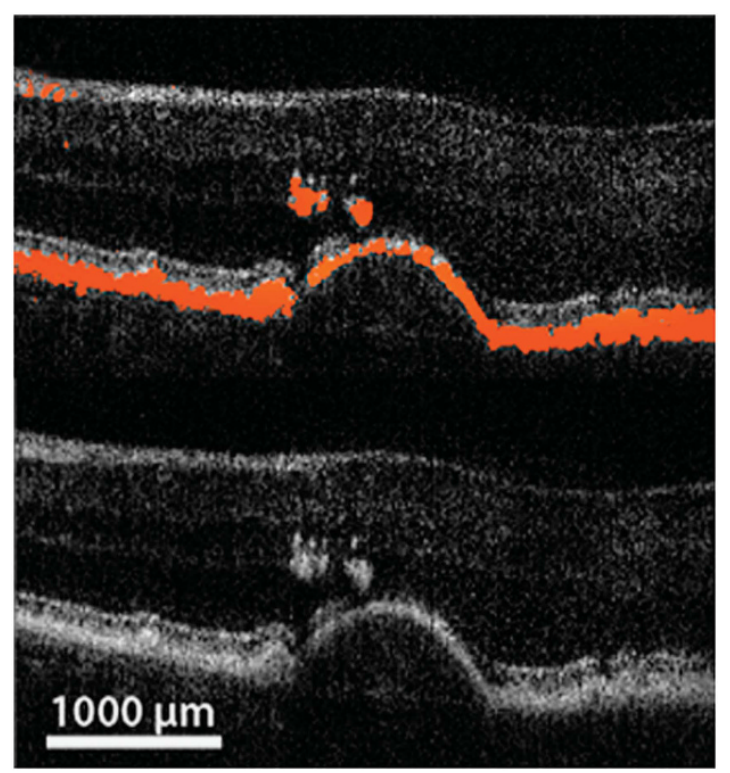

Figure 4 Example of macular drusen characteristics visualized by polarization-sensitive OCT. Upper part of the image shows depolarization signal in red, while the conventional intensity image as in SD-OCT is presented in the lower part. A large druse with overlying hyper-reflective foci is visualized. (Courtesy of Schlanitz et $a l^{32}$ ). regimens on an individual as well as on a populationbased level. ${ }^{35}$

\section{Features of pathognomonic value}

Following the introduction of OCT into everyday clinical practice, Freund et al ${ }^{36}$ developed a classification of $\mathrm{CNV}$ according to its location, combining fluorescein angiography (FA), OCT, and-if needed-ICGA in a multimodal imaging approach. In OCT, type $1 \mathrm{CNV}$, located between BM and RPE, corresponds to PED, often accompanied by subretinal fluid and in later stages of disease by intraretinal cystoid fluid. Type $2 \mathrm{CNV}$ presents as subretinal hyperreflective material (SHRM) and exhibits concomitant intraretinal cystoid fluid and subretinal fluid. ${ }^{36}$

PED can present as fibrovascular or serous, and OCT allows reliable characterization of these entities.

Fibrovascular PED, constituting type $1 \mathrm{CNV}$, appears as irregular RPE elevation with or without serous exudation. ${ }^{33}$ The CNV membrane itself has been shown to correspond to hyperreflective material along the back surface of the PED, readily visible by enhanced-depth imaging, ${ }^{37}$ or a tomographic notch within the PED, identifiable by conventional OCT. ${ }^{38}$ Serous PED, in most cases with neovascular AMD, presenting the correlate of leakage from type $1 \mathrm{CNV}$, appears as a smooth, sharply demarcated, and dome-shaped elevation of the RPE overlying a homogenous hyporeflective space (Figure 6a). ${ }^{33}$ Mostly assumed to be avascular, serous PED, however, can also be partially vascularized.

Detection of the neovascular membrane becomes feasible with enhanced depth imaging or by use of OCT angiography. Kuehlewein et $a l^{39}$ visualized well-defined neovascular complexes in $75 \%$ of eyes presenting with PED (Figure 5). Although no statistically significant correlation could be detected between vessel pattern and PED subtype or the previous frequency of anti-VEGF injections, further investigations might give a more detailed insight, in particular considering alterations of the choriocapillaris as a pathomechanism for disease. ${ }^{39}$ Bearing in mind that PED often presents inherent CNV, a reduced treatment response of PED compared with intraretinal cystoid fluid and subretinal fluid seems plausible, as therapy targets exudation rather than the neovascular lesion itself. ${ }^{40,41}$ Nor does it surprise that serous PED occasionally has been shown to respond best. ${ }^{42}$ No statistically significant change in area or vessel density after treatment with anti-VEGF therapy has been found in OCT angiography of PEDs. ${ }^{39}$ Enhanced depth imaging has revealed hyperreflective material to be present in all PEDs, regardless of their optical emptiness in conventional OCT imaging. ${ }^{37}$ The further implications of these findings still need to be evaluated. 
Subretinal fluid, as the predominant form of fluid exudation in type $1 \mathrm{CNV}$, can be clearly characterized as hyporeflective fluid accumulation overlying the RPE layer (Figures $6 \mathrm{a}$ and $\mathrm{b}$ ). It resolves in most eyes in response to anti-VEGF treatment, however, not as rapidly as intraretinal cystoid fluid. ${ }^{41}$ Intraretinal cystoid fluid mostly appears as round or oval hyporeflective spaces (Figure 6a), but diffuse thickening of the neurosensory retina, without clear borders, may also present intraretinal cystoid fluid, raising difficulties in identification and quantification. Although reports usually associate presentation of intraretinal cystoid fluid with active fluid exudation, appreciation of degenerative cystoid fluid, thought to derive from passive fluid accumulation due to atrophy of neurosensory elements, should not be underestimated. ${ }^{35}$ Difficult to distinguish by appearance on OCT, degenerative intraretinal cystoid fluid is better characterized by its response to anti-VEGF treatment. Although exudative intraretinal cystoid fluid typically shows rapid and pronounced resolution during the loading phase, degenerative cysts do not respond markedly during this phase. The highly dynamic response of exudative intraretinal cystoid fluid to antiVEGF treatment applies also to treatment-free periods and has therefore been shown to result in recurrent increases in fluid presentation between treatments. ${ }^{41}$
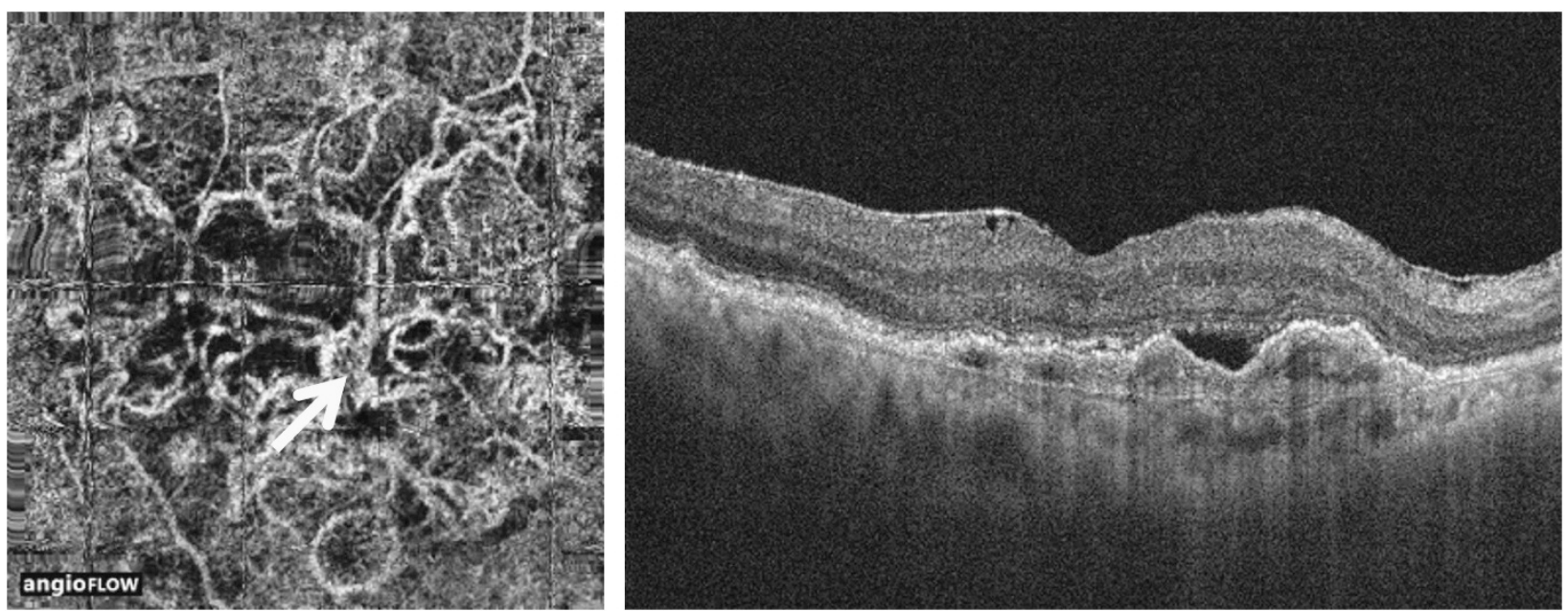

Figure 5 Type 1 CNV in neovascular AMD, visualized by OCT angiography. Left image shows OCT angiography visualization of the neovascular complex. Right image shows conventional intensity image of a fibrovascular PED harboring CNV. (Figure from Kuehlewein et $\left.a l^{39}\right)$.
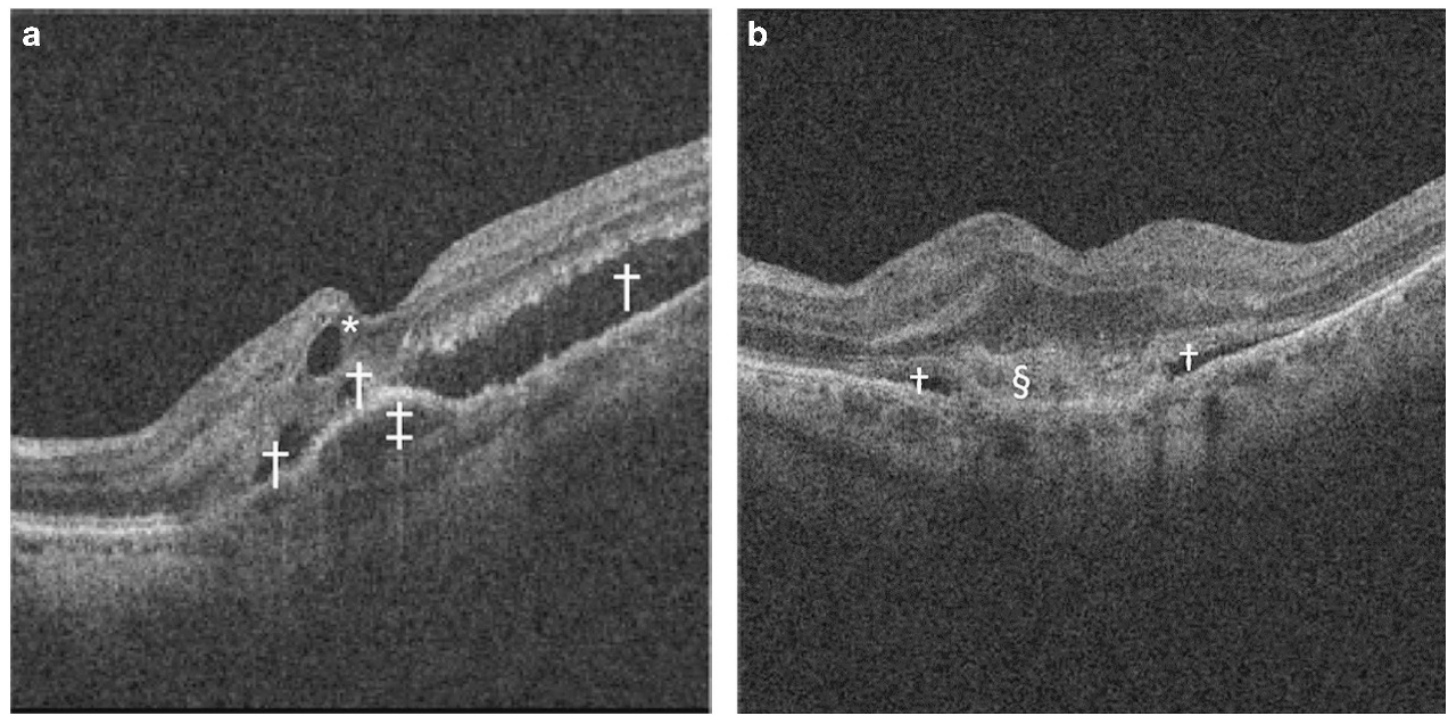

Figure 6 SD-OCT features of neovascular AMD. (a) Macular SD-OCT scan presenting serous PED ( $\ddagger$ ), subretinal fluid ( $†$ ), and intraretinal cystoid fluid (*). (b) Macular SD-OCT scan presenting subretinal fluid alongside SHRM (§). 
Intraretinal cystoid fluid overlying PED, accompanied by subretinal fluid, are typical features commonly present in retinal angiomatous proliferation (RAP), classified as type $3 \mathrm{CNV}$ by Freund et al. ${ }^{36} \mathrm{RAP}$ presents retinochoroidal anastomoses, which may form in advanced disease or primarily. Querques et $a l^{43}$ described progressive stages of RAP, first the eroding of the RPE (phase 1), later the breaking through, infiltrating first into the outer retina (phase 2) and further into the inner retina (phase 3), forming the 'kissing sign', which presents a focal funnel-shaped RPE joining an inverted focal funnelshaped inner neuroepithelium. Mature type 3 lesions, associated with serous PEDs, are highly responsive to anti-VEGF therapy. ${ }^{44}$ However, the development of GA has frequently been described in association with treatment of RAP lesions. ${ }^{45}$ With OCT angiography, qualitative and quantitative analysis of the anastomotic neovascular complex has been shown to be feasible. ${ }^{46}$

Type 2 CNV is accompanied by SHRM, an amorphous lesion of medium-to-high reflectivity, located in the subretinal space, external to the retina and internal to the RPE (Figure 6b). ${ }^{33}$ SHRM, however, can also comprise fluid exudation, fibrin, blood, or scarring $33,47,48$ and its composition changes over time during therapy. ${ }^{49}$ In a post-hoc analysis of the Comparison of Age-Related Macular Degeneration Treatment Trials (CATT), SHRM was shown to present in $77 \%$ of treatment-naive eyes at baseline with the prevalence decreasing to $58 \%$ at week 4 after treatment and further to $46 \%$ after 2 years, probably owing to a dehydration and condensation of the active CNV component. ${ }^{49}$ OCT angiography and polarizationsensitive OCT are promising tools for identifying the inherent components of SHRM. OCT angiography allows a distinction between vascular and avascular constituents by assessment of intrinsic flow. However, inner retinal vessel projection onto the SHRM surface as well as the RPE and masking of choriocapillaris flow have to be considered. Despite the lack of masked observer studies confirming the ability of OCT angiography to characterize SHRM, identification of type $2 \mathrm{CNV}$ using this imaging modality seems promising. ${ }^{50}$ In addition, polarizationsensitive OCT offers selective identification of fibrous tissue within SHRM based on its birefringence, visualized as distinct column-like patterns within an otherwise unstructured lesion. ${ }^{47}$

The role of hyper-reflective foci as an important feature in assessing progression from early-to-late AMD has been described in the section on early and intermediate AMD. In treatment-naive patients with exudative AMD, hyperreflective foci have been shown to be present to an at least moderate extent in $>75 \%$ of eyes. This feature of exudative disease also shows statistically significant reduction during anti-VEGF therapy. ${ }^{51-54}$

\section{Risk of progression}

Several studies have revealed the very limited suitability of central retinal thickness as a basic retinal feature for disease assessment owing to the lack of its correlation with visual acuity (VA) during follow-up. ${ }^{41,55}$ Detailed qualitative and quantitative analysis of the abovementioned pathognomonic features, among others, is, therefore, crucial for insight into disease mechanics and treatment efficacy.

Intraretinal cystoid fluid is to date the most important predictor of visual function at baseline as well as visual outcome following anti-VEGF therapy, regardless of whether treatment is frequent or infrequent, with patients presenting with intraretinal cystoid fluid being at a functional disadvantage. ${ }^{40,41,55-59}$ Although a post-hoc analysis of the Efficacy and Safety of Monthly vs Quarterly Ranibizumab Treatment in Neovascular Age-related Macular Degeneration (EXCITE) study revealed no further negative impact of intraretinal cystoid fluid recurrence per se on $\mathrm{VA}^{41}$ patients who showed degenerative cysts, defined as cysts that have not disappeared after the loading phase, presented with an even lower visual function. ${ }^{40}$ Patients with intraretinal cystoid fluid still remaining after 12 monthly anti-VEGF injections have further shown a higher risk for fibrosis and RPE atrophy compared with patients presenting refractory SRF. ${ }^{60}$ Development of GA has also been associated with the presence of intraretinal cystoid fluid at the foveal center at baseline. ${ }^{45}$

By contrast, PED appears to be a pathomorphological component associated with lower VA only under rare circumstances. Despite reported lower VA at baseline, ${ }^{55}$ PED has a negative effect on visual function only in combination with additional components, mostly intraretinal cystoid fluid. ${ }^{41,55}$ Most importantly, the type of treatment regimen is an important factor when considering the role of PED for functional outcomes. A post-hoc analysis of the VEGF Trap-Eye: Investigation of Efficacy and Safety in Wet Age-Related Macular Degeneration (VIEW) trials found the switch from a monthly to an as-needed, pro re nata (PRN) regimen led to reactivation of PED with a resultant decline in visual function, especially in patients who developed secondary intraretinal cystoid fluid following this event (Figure 7). ${ }^{40}$ So far, PED has not been regarded as a relevant imaging marker for PRN regimens. It seems, however, that recurrence of PED is the primary event of neovascular activation, with secondary intraretinal cystoid fluid being mainly responsible for irreversible functional loss. ${ }^{40}$ A small retrospective study of automated volumetric analysis of PED found that PED growth seemed to precede retreatment and, therefore, fluid recurrence. ${ }^{61}$ Analysis of the correlation between VA and the types of 
PED revealed a slight trend toward better VA with serous PED. ${ }^{62}$ OCT analysis of PEDs may distinguish those of risk in developing an RPE tear. Large PEDs $>550 \mu \mathrm{m}$ present an increased risk after the first anti-VEGF treatments in eyes where the sub-RPE CNV has created contractile folds in response to treatment. ${ }^{63,64}$

Subretinal fluid is an imaging biomarker showing a stable association with better visual outcomes regardless of treatment frequency. ${ }^{40,42,57,59,65}$ Among patients treated with a PRN regimen, those who present with subretinal fluid achieve even higher VA gains (Figure 8). ${ }^{59}$ So far, the pathomechanism for the beneficial role of subretinal fluid has not been fully explained. Reduced rates of concomitant RPE atrophy and fibrosis as well as the preservation of photoreceptor integrity and signs indicating a possible exclusion of vision-deteriorating intraretinal cystoid fluid at the same retinal location have been suggested as explanations (Sharma et al ${ }^{57}$; Gianniou et $a l^{60}$; Philip et al submitted; Klimscha et al submitted). Analysis by microperimetry has shown higher improvement rates in retinal sensitivity for subretinal fluid and serous PED than for intraretinal cystoid fluid and fibrovascular PED. ${ }^{66}$ Further, refractory subretinal fluid present despite 12 months of anti-VEGF injections has shown no detrimental effects on visual outcome. ${ }^{60}$

The condition of the vitreomacular interface seems to affect treatment outcome in correlation with the presence of subretinal fluid. Posterior vitreomacular detachment has been associated with a more benign disease course even with infrequent (quarterly) or PRN treatment regimens. ${ }^{59,67,68}$ This effect seems to be augmented by the concomitant presence of subretinal fluid. ${ }^{59}$ Vitreomacular adhesion, on the other hand, shows excellent VA outcomes with a fixed treatment regimen while infrequent or PRN treatment leads to a less favorable prognosis in eyes with vitreomacular adhesion. ${ }^{67,68}$

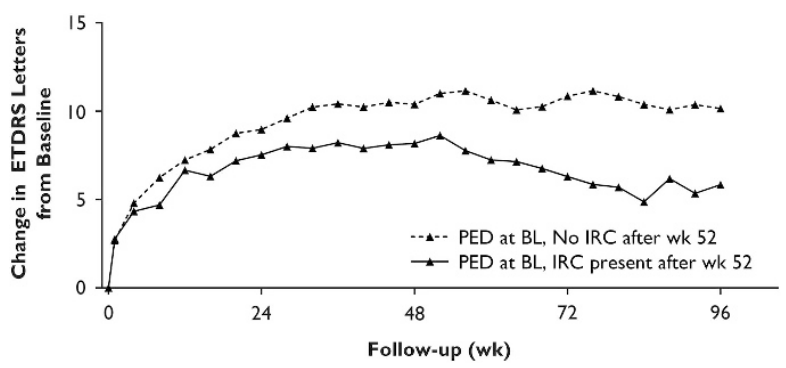

Figure 7 Change in visual acuity in patients with PED at baseline (BL) by intraretinal cystoid fluid (IRC) status in the VIEW2 study. Note the vision loss in patients with PED at BL owing to secondarily developing IRC after the switch to a PRN treatment regimen at week 48. ETDRS, Early Treatment Diabetic Retinopathy Study. (Figure modified from Schmidt-Erfurth et $\left.a l^{40}\right)$.
Pharmacokinetic differences and lower cytokineincluding VEGF-levels have been suggested as reasons for the beneficial role of a posterior vitreomacular detachment. ${ }^{67,69}$

The presence and location of hyperreflective foci as a predictor of anatomic and functional changes in antiVEGF therapy has been studied intensively. To date, only one study has reported resolution of hyperreflective foci to be associated with better visual outcomes. ${ }^{70}$ Several investigators have found their resolution solely to be associated with reduction in central retinal thickness, 51,54 which per se has shown to lack correlation with visual function during the maintenance phase. Analyses of the impact of the mere presence and location of hyperreflective foci on visual function at baseline and during therapy have revealed that a high amount $(>20)$ of hyperreflective foci and a location in the inner and subretinal layers are associated with worse VA outcomes. $^{52-54}$

Formation of a disciform scar via degradation and remodeling of extracellular space is the ultimate consequence of an untreated or treatment-resistant neovascular invasion, accompanied by loss of RPE and photoreceptors. ${ }^{33}$ Photoreceptor integrity, naturally, is vital for visual improvement during anti-VEGF therapy. Analyses of alterations of the outer retinal layers preceding the development of irreversible vision loss may provide an insight into the pathomechanisms of exudative disease. The power of previous investigations has, however, been limited by the laborious quantification and poor reproducibility of the changes identified in the outer retinal bands. ${ }^{35}$ Nevertheless, in an observation that lasted a minimum of 12 months after initiation of antiVEGF therapy, the initial preservation of a foveal ELM was shown to be correlated with better final VA. ${ }^{52}$ A retrospective study with a mean follow-up of 6 months showed that the length of disruption of the EZ at baseline

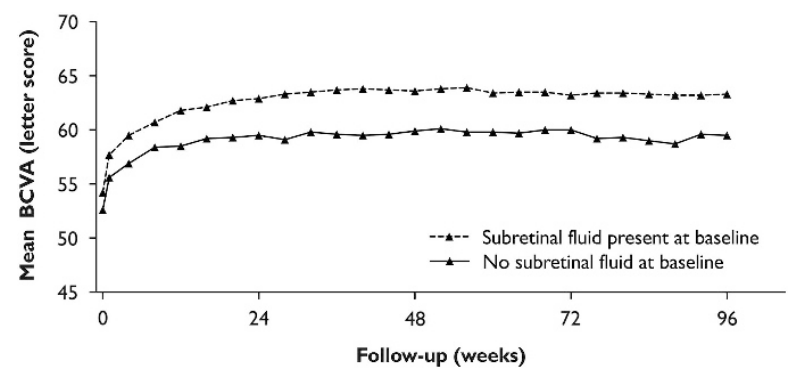

Figure 8 Development of best-corrected visual acuity (BCVA) dependent on the presence of subretinal fluid at baseline in the VIEW trial. Eyes with subretinal fluid consistently show higher visual improvement rates. (Figure modified from Waldstein et $\left.a l^{65}\right)$. 
correlated positively with the final VA. ${ }^{53}$ Further, a posthoc analysis of CATT found the EZ was absent in the foveal center more often in eyes with underlying foveal SHRM. ${ }^{49}$ However, analysis of the ELM alone did not reveal similar results. SHRM, as described previously, can be the correlate of various pathologies, and its presence as well as increased height and width have been associated with worse VA at baseline and during therapy. ${ }^{49,57}$ Further, its resolution seems to entail superior VA improvement. The presence and development of scarring - evaluated by color fundus photography and FA-was increased for patients with persisting SHRM. Whether a distinction between SHRM and the RPE layer was possible did not influence VA. ${ }^{49}$

RPE atrophy develops during natural disease progression in neovascular AMD as well as during antiVEGF therapy. An enhancing effect of anti-VEGF agents on atrophy development has been suspected and controversy persists because characterization and quantification of this effect is difficult. ${ }^{33,35}$ Assessment of RPE atrophy by OCT has been described in the section on early and intermediate AMD. In neovascular disease, RPE atrophy, sometimes accompanied by atrophy of the overlying neurosensory retina, seems to develop predominantly in areas previously occupied by CNV. ${ }^{71}$ Eyes with type $1 \mathrm{CNV}$ are less likely to develop GA. ${ }^{72}$ The prevalence of RPE atrophy among eyes with neovascular AMD has varied greatly in reports, mainly owing to discrepancies in definition and imaging modalities used for identification. Atrophy in the CATT trial, which was assessed by color fundus photography and FA, was reported to be $10-25 \%$ at years 1 and 2 and $41 \%$ at year 5 while Abdelfattah et $a l^{73}$ reported atrophy, as graded by OCT, to be already present in $46 \%$ of eyes at baseline, all of which showed progression in atrophy during follow-up.

\section{Patient management}

The diagnosis and management of neovascular AMD was revolutionized by the introduction of OCT into everyday clinical practice. Despite the ability of OCT to enable a confident diagnosis via assessment of various pathognomonic features of disease, FA, which was formerly required for primary diagnosis, is still being performed at first presentation. ${ }^{33}$ In terms of disease monitoring and evaluation of treatment efficacy, OCT has evolved to become the core assessment. Not only is it suitable for providing information about individual prognosis but also, more importantly, retreatment criteria in PRN regimens are based mostly on OCT-based features. In general, it can be said that the efficacy of antiangiogenetic therapy highly correlates with resolution of morphological features. ${ }^{35}$
Intraretinal cystoid fluid is the main OCT-detectable feature that indicates poorer VA outcome. Further, patients with a large extent of SHRM or damage to the photoreceptor or RPE layer are counseled that their VA gains will most likely not reach that of the average patient. The inferior visual outcome of PRN treatment regimens ${ }^{34}$ can be attributed to the treatment, regardless of the underlying pathomorphological activity. Several features on OCT have proven to present signs of persistent disease, requiring frequent treatment and monitoring. First and foremost, exudative intraretinal cystoid fluid has been shown to require monthly treatment, in particular after recurrence of PED, which seems to harbor the potential of disease reactivation via its primary disease component. ${ }^{40}$ Further, if vitreomacular adhesion is present, monthly treatment is needed to achieve ideal visual gains.

PED, in principle believed to have a benign influence on disease course via compensatory mechanisms, ${ }^{36}$ has been shown to reactivate after a switch from a monthly to flexible treatment regimen. Therefore, PED should be included in retreatment recommendations of PRN treatment regimens in future. Currently, the recommendations only take into account changes in VA or central retinal thickness as well as the presence of intraretinal or subretinal fluid or qualitative changes thereof. ${ }^{40}$ By contrast, subretinal fluid is associated with stable VA, regardless of treatment frequency, and with overall higher visual outcomes. ${ }^{40,42,57,59}$ Consequently, subretinal fluid is an ideal feature for identifying patients suitable for flexible or treat and extend regimens.

It should, however, be noted that all the above conclusions and suggestions are based on retrospective observations. Although some of the retrospective analyses are based on large prospective randomized trials, none of these trials were designed to assess the correlation between morphology and treatment efficacy.

Nevertheless, many of the observations are likely to be valid because they have been consistent and corroborative. Prospective studies investigating and confirming morphological implications would be of great value for ideally tailoring flexible antiangiogenetic treatment regimens. To our knowledge, the Comparison of Treatment Regimens Using Ranibizumab: Intensive (Resolution of Intra- and Sub-retinal Fluid) vs Relaxed (Resolution of Primarily Intra-retinal Fluid) Treatment (FLUID) study, conducted across multiple centers in Australia between 2013 and 2015 and with results still outstanding, is the only investigation to compare different retreatment criteria based on morphology on a more differentiated level. ${ }^{74}$

OCT angiography, when used in a multimodal approach, has the potential to provide essential additional information on anatomic, angiogenic correlates and the 
treatment response of features seen on conventional OCT. Active CNV lesions may be differentiated from stable fibrous complexes in the case of SHRM, which would potentially be relevant for treatment decisions.

\section{Future perspectives and technology}

As OCT provides a large amount of information of the retina, manual analysis of pathognomonic features of $\mathrm{CNV}$ is difficult and time consuming, limiting it only to coarse qualitative aspects (e.g., presence or absence of a feature). Consequently, computational image analysis pipelines are needed to provide precise quantitative measurements of the features in an automated, objective, and repeatable way. Recently, algorithms have been proposed for the analysis of OCT images with CNV, namely multi-resolution graph-theoretic-based surface detection for PED segmentation ${ }^{75,76}$ and machine learning-based pixel classification for intraretinal cystoid fluid and subretinal fluid segmentations. ${ }^{77,78}$ The accuracy of such automated quantification is expected to improve further in the near future and come within interobserver/reader variability, making results of automated algorithms indiscernible from reader annotations.

An important clinical application of interest is to predict treatment response as soon as possible and estimate the success of the planned regimen. Being able to process and segment individual OCT scans in an automated manner allows clinicians to use large longitudinal data sets, where each patient has a baseline and multiple follow-up images acquired. Furthermore, such data sets can be mined for predictive imaging biomarkers. Anti-VEGF treatments start with a loading phase, during which patients are treated in a standardized way. Such uniformity of treatment allows clinicians to learn the retinal response and differentiate between patients and their later treatment success based on the longitudinal time course of their quantitative morphological features. Machine learning algorithms are particularly suitable for ascertaining treatment outcome from the loading phase, achieving good predictions, and revealing the predictive features. Such approaches have recently been used to predict whether a patient with $\mathrm{CNV}$ will respond to anti-VEGF treatment ${ }^{79}$ and whether macular edema will recur. ${ }^{80}$ Computational analysis of OCT images is expected to become even more widespread in the coming years. This will hopefully lead to a set of powerful tools that will allow personalized anti-VEGF treatments to be created by identifying subtle, but important, differences in retinal responses between patients.

\section{Advanced atrophic AMD (GA)}

GA is the second manifestation of late AMD next to exudative disease and is characterized by a more gradual, but nevertheless ultimately significant, loss of vision. Drusen- and neovascularization-associated GA have been discussed in previous sections. Detection of GA has been facilitated by various imaging techniques, FAF being the gold standard. GA measurements on OCT have, however, been shown to correlate well with FAF measurements and, further, to have advantages including the assessment of the central foveal area. The cross-sectional properties of OCT images allow subtle changes in the outer retinal layers at GA margins to be visualized. ${ }^{33}$

\section{Features of pathognomonic value}

GA identification on SD-OCT is based on the characteristic hypertransmission of light into the choroid below the BM, which is due to the absence of lightscattering RPE and varying degrees of choriocapillaris (Figure 9). Confluent areas of RPE atrophy are further accompanied by the loss of the overlying photoreceptors, absence of the ELM, thinning, loss or subsidence of the ONL, and subsidence of the OPL. Regressing drusen, residual, dotted drusen material, and irregular elevations of the remaining part of the RPE-BM complex may be identified (Figure 9c). ${ }^{33,81,82}$ En-face projections of the summed transmitted and reflected light from all layers, named OCT fundus images, offer additional visualization. GA appears brighter than the surrounding areas on OCT fundus images owing to the increased total signal from the choroid. Manual and automated quantification of the GA area using OCT fundus images has been shown to be valid, reproducible, and useful in assessing GA growth. ${ }^{82}$ Sub-RPE slab images solely use light that is reflected back from the choroid beneath the $\mathrm{BM}$ and, therefore, tend to show higher contrast at the margins of GA. ${ }^{82}$ This approach is being applied in the only commercially available, fully automated FDAapproved GA segmentation algorithm, which has been integrated into Cirrus HD-OCT instruments.

Borders of GA are defined in OCT, as the change from a hyporeflective to hyperreflective choroidal signal. Various morphological alterations of these junctional zones, solely identifiable on OCT, have been observed. Fleckenstein et $a^{81}$ described abrupt, regular breaks in the EZ, the interdigitation zone (IZ), and the RPE, three of the four outer retinal bands as well as irregular disruptions obscuring the delineation of these bands. The ELM often presents as a line downwardly curved to merge with the atrophy.

GA is a dynamic condition with its area constantly enlarging in all patients (Figure 10). ${ }^{82,83}$ Progressive 
a

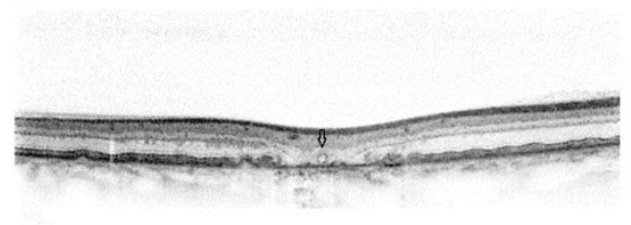

b

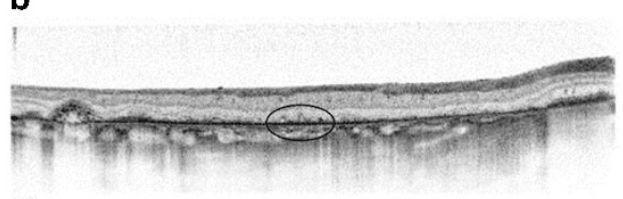

C

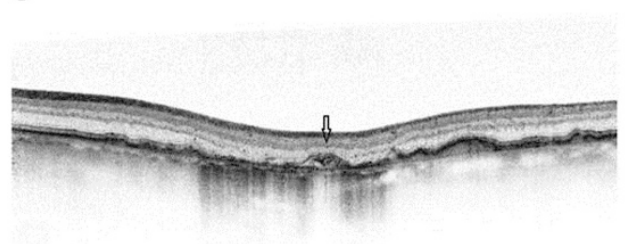

d

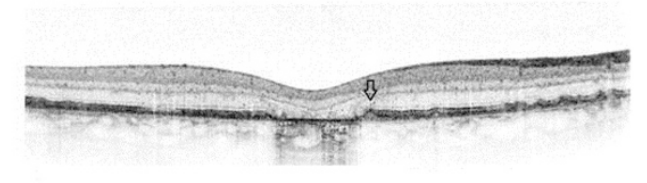

e

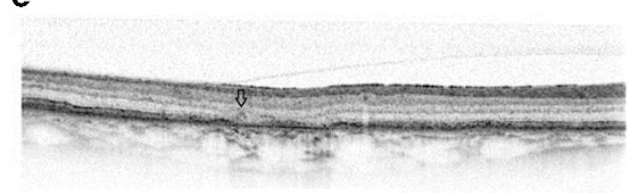

f

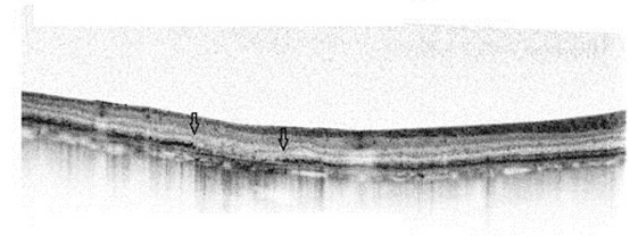

Figure 9 Microstructural alterations in the region of GA and junctional zones, visualized by SD-OCT. GA appears as a loss of RPE and presents a hyper-reflective signal in the choroid. Arrows/circle mark outer retinal tubulation (ORT) in panels (a and e), irregular elevations of the RPE-BM complex in panel (b), crown-like elevations with debris beneath in panel (c), dome-shaped RPE elevation in panel (d), and splitting of the RPE-BM complex in panel (f).

expansion of the loss of RPE, photoreceptors, and varying degrees of choriocapillaris characterizes the process of enlargement. Yehoshua et al ${ }^{82}$ manually quantified GA on OCT fundus images with a minimum follow-up of 6 months in a prospective natural history study and detected a mean GA enlargement rate of $1.2 \mathrm{~mm}^{2} /$ year and $0.28 \mathrm{~mm} /$ year, applying a square root transformation. Previous studies that assessed GA progression on $\mathrm{FAF}^{83}$ using the area scale found that enlargement rates correlated with baseline lesion size, with larger lesions growing at a faster rate. Square root transformed data, however, showed no correlation between enlargement and baseline area and is, therefore, suitable for assessment of GA in clinical trials testing treatment efficacy as baseline lesion size can be neglected as an inclusion criteria. That the enlargement rate was lower as assessed by OCT than in previous studies applying FAF imaging ${ }^{83}$ can be explained by the smaller mean lesions sizes at baseline as well as differences in the pathomorphological correlates of GA imaged. Threequarters of the lesions in 86 eyes of the 64 patients were classified as multifocal and one-quarter as unifocal, with multifocal lesions presenting smaller mean areas at baseline. Stratification revealed a significant difference in enlargement rate on the square root scale, with a mean rate of $0.32 \mathrm{~mm} /$ year in multifocal and $0.19 \mathrm{~mm} /$ year in unifocal lesions. ${ }^{82}$ High intraocular variability of enlargement between inferior, superior, nasal, and temporal borders of GA has been observed. In addition, morphological changes within the atrophic region have been observed over time. The irregular structure of the remaining RPE-BM complex became more homogenous with time. A thin band representing the BM remained in all eyes. ONL showed progressive thinning, resulting in the OPL further approaching the BM. ${ }^{84}$

Areas of choroidal hyper-reflectivity, OPL thinning, and ELM loss identified on OCT correlate well with GA planimetry on FAF, based on hypoautofluorescence. ${ }^{85}$ Foveal sparing, however, which is often observed in GA, is best visualized by OCT. FAF imaging encounters difficulties in identifying exact GA boundaries in the foveal region owing to absorption of excitation light by xanthophylls, which further block the excitation of lipofuscin-containing RPE. ${ }^{26,83}$ Mild retinal swelling, attributed to the ONL, has been described on OCT in these areas of foveal sparing and may present preapoptotic stages preceding atrophy. 86

\section{Risk of progression}

Identification of the risk factors, besides baseline lesion size, for the growth and growth rate of GA are highly 

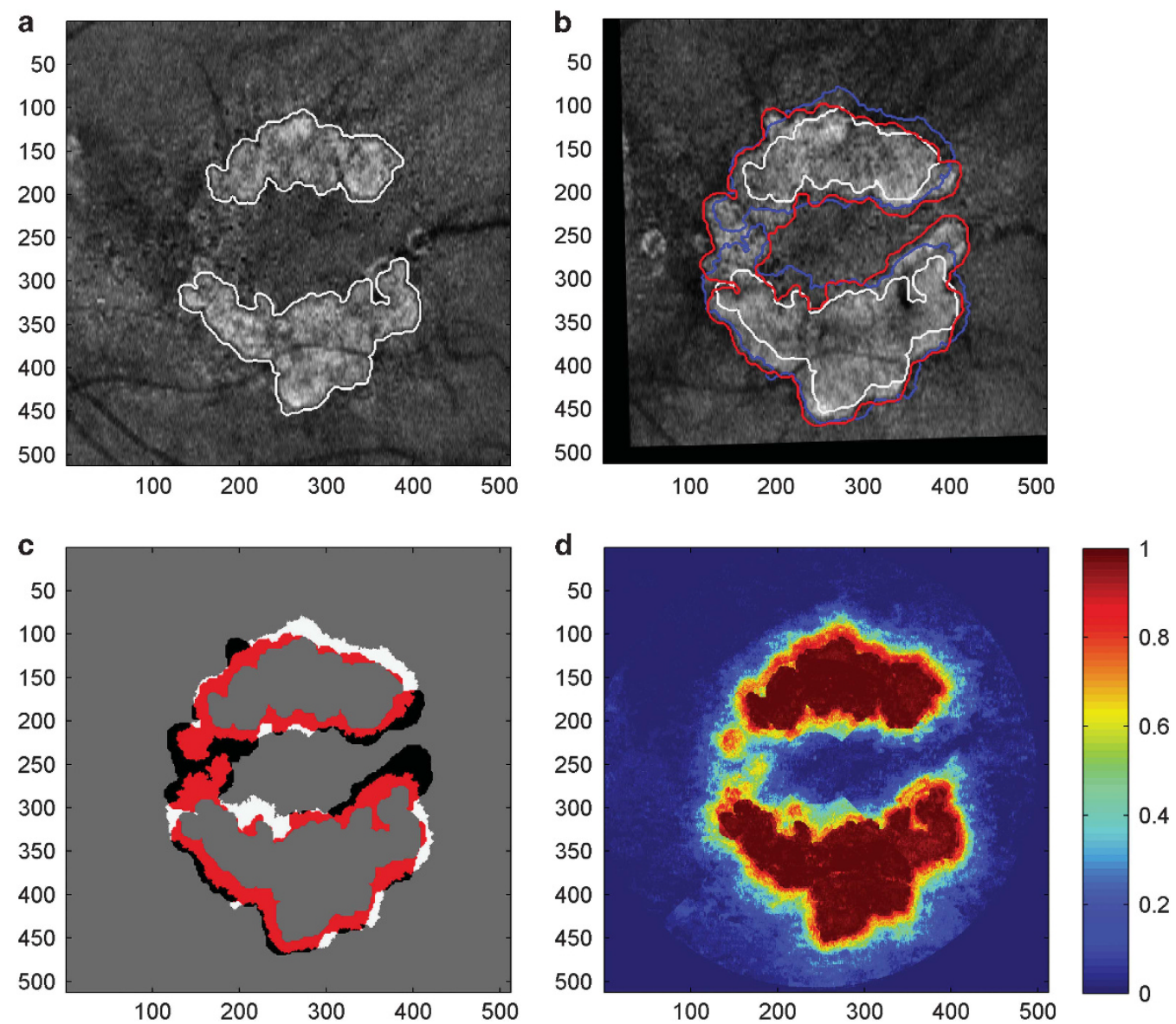

Figure 10 Fully automated prediction of GA growth from a baseline observation. (a) OCT fundus image of GA at baseline with automatically segmented GA outlined in white. (b) OCT fundus image of GA obtained 12 months from baseline. Baseline GA region shown in white, observed GA region at follow-up shown in red, predicted GA region shown in blue. (c) Comparison of predicted and observed results. The red and white regions depict the correctly predicted (true-positive) and incorrectly predicted (false-positive) regions, respectively. Black regions correspond to false-negative results. (d) Classification probability obtained from the evaluation of the baseline scan. (Figure from Niu $\mathrm{et}_{\mathrm{al}}{ }^{98}$ ).

sought after as GA first appears in the parafoveal location, moving through the fovea only as time progresses. Changes in the outer retinal layers, most importantly, photoreceptor abnormalities, can precede the enlargement of GA and may serve as early indicators of disease progression. In a prospective observation of GA progression, Fleckenstein $e t$ al ${ }^{84}$ observed severe alterations in all of the outer retinal bands as well as ONL thinning in most eyes at junctional zones, where RPE loss and choroidal hyper-reflectivity had not yet occurred, which suggests that photoreceptor loss often extends beyond the borders of GA. Research interests have, therefore, focused on investigations of changes within the junctional zones, especially the photoreceptor layer.

Knowledge of these changes is expected to give a deeper understanding of disease pathogenesis. A small prospective case series designed to determine whether photoreceptor loss or RPE loss initiated GA found photoreceptor loss most frequently appeared as bridging across the GA margin, less frequently within the GA margin, and least frequently entirely outside the GA margin, thus not conclusively isolating the initiating event. ${ }^{87} \mathrm{~A}$ statistically significant increase in minimum OCT scan intensity has been shown in areas of GA growth, possibly presenting disruption or loss of photoreceptors. Prediction of GA growth on the basis of minimum intensity seems feasible. ${ }^{88}$ Outer retinal tubulations are branching tubular structures appearing as round or ovoid hyporeflective spaces with hyperreflective borders located in the ONL. Outer retinal tabulations and irregular elevations of the remaining RPE-BM complex in atrophic areas as well as splitting of the RPE/BM complex at GA borders have been correlated with faster enlargement rates (Figure 9). ${ }^{89}$

Further, irregular thickening as well as splitting of the RPE-BM complex have been observed at GA margins. ${ }^{90}$ 
Later, they were found to correspond with areas of increased autofluorescence at the margin of GA, imaged with FAF. ${ }^{90,91}$ Different patterns of autofluorescence have been correlated with varying risks of enlargement, eyes with a banded or diffused hyperautofluorescence pattern at GA margins showing statistically significantly higher enlargement rates than those without FAF abnormalities. Diffuse trickling, a subtype of perilesional hyperautofluorescence associated with an even higher enlargement rate, has been shown to more likely present splitting of the RPE-BM complex at GA borders. ${ }^{89}$ Hyper-reflective foci were found in the perilesional zones of all eyes. Dynamic changes in hyperreflective foci during follow-up involved an increase in vertical extension with penetration of EZ and ELM as well as migration toward inner retinal layers. Drusen were present in the perilesional areas of most eyes. Dynamic changes of drusen, described in the section of early and intermediate AMD, including an increase and decrease in drusen height, occurred frequently, with a decrease often preceding the development of atrophy. Additionally, a median change in retinal thickness of $-14.09 \mu \mathrm{m}$ /year was observed at the borders of GA. A decrease in retinal thickness mostly correlated with a loss of outer retinal layers. Retinal thickness changes, particularly increases, can be influenced by the development of epiretinal membranes and RPE elevations or sub-RPE deposits and should, therefore, be interpreted with caution. ${ }^{84,87}$ Multifocal compared with unifocal GA lesions seem to entail a risk of higher enlargement rates. ${ }^{82}$

\section{Patient management}

OCT is reliable for visualizing and quantifying GA. ${ }^{82}$ Its exceptional benefits include the visualization of loss of photoreceptors and demarcation of GA margins in the central foveal area. Owing to the lack of an approved or effective treatment for the late atrophic stage of AMD, clinical use of OCT can currently only serve the purpose of diagnosis, characterization, and prediction of disease progression. However, Schaal et $a l^{26}$ in their recent review of clinical end points in non-exudative AMD highlighted the important role of OCT in the design of valid surrogate markers of disease progression, as visual function does not relate well to disease progression. Reliable and reproducible quantification of GA using OCT fundus images and sub-RPE slabs, and, further, applying square root transformations in the analysis of data sets, generate suitable surrogate end points for the clinical trials and investigational therapies currently underway. ${ }^{26}$ Further investigation of OCT-detectable features within and at the margins of GA might provide biomarkers that correlate to even earlier changes in the pathogenesis of GA.

\section{Future technology and perspectives}

Ultrahigh-speed, swept-source OCT angiography has rendered visualization of choroidal flow possible and thereby shown pronounced choriocapillaris flow impairment within the regions of GA. Non-invasive assessment of alterations in retinal and choriocapillaris vasculature in eyes with GA appears to be a promising technology for investigating disease pathogenesis and progression and the response to therapy. ${ }^{92}$ Polarizationsensitive OCT, a further new, evolving imaging modality, has been shown to offer highly reproducible measurements of GA lesion size, similar to FAF and OCT fundus images, based on tissue-specific depolarizing properties of the RPE. ${ }^{93}$ Polarization-sensitive OCT allowed the most accurate assessment of the fovea in a study comparing foveal integrity in GA between different imaging modalities and visual function. ${ }^{94}$ The advantages and disadvantages of various imaging modalities in grading GA need to be taken into account for diagnostic and therapeutic evaluations as well as future clinical trials.

Various automated ${ }^{95}$ and semiautomated 96,97 algorithms measuring and quantifying areas of GA have been developed with the aim of assisting the laborious effort of manual GA assessment and streamlining analysis of large data sets. Advances in computational analysis and machine learning have made the prediction of future potential regions of GA growth, enlargement rate, and foveal involvement possible. Recently, Niu et al ${ }^{98}$ applied a fully automated pipeline for OCT segmentation, feature extraction, predictive modeling via machine learning, and testing of methods (Figure 10). They presented experimental results predicting GA progression with a good performance based on quantitative features, characterizing GA extent and location as well as individual retinal layer thickness and reflectivity. Further, they identified photoreceptor loss as the most discriminant early indicator of regions susceptible to GA growth.

\section{Conflict of interest}

The authors declare no conflict of interest.

\section{Acknowledgements}

Financial support by the Austrian Federal Ministry of Science, Research and Economy and the National Foundation for Research, Technology and Development is gratefully acknowledged. 


\section{References}

1 Ferris FL 3rd, Wilkinson CP, Bird A, Chakravarthy U, Chew E, Csaky $\mathrm{K}$ et al. Clinical classification of age-related macular degeneration. Ophthalmology 2013; 120(4): 844-851.

2 Farsiu S, Chiu SJ, O'Connell RV, Folgar FA, Yuan E, Izatt JA et al. Quantitative classification of eyes with and without intermediate age-related macular degeneration using optical coherence tomography. Ophthalmology 2014; 121(1): 162-172.

3 Khanifar AA, Koreishi AF, Izatt JA, Toth CA. Drusen ultrastructure imaging with spectral domain optical coherence tomography in age-related macular degeneration. Ophthalmology 2008; 115(11): 1883-1890.

4 Leuschen JN, Schuman SG, Winter KP, McCall MN, Wong WT, Chew EY et al. Spectral-domain optical coherence tomography characteristics of intermediate age-related macular degeneration. Ophthalmology 2013; 120(1): 140-150.

5 Spaide RF, Curcio CA. Drusen characterization with multimodal imaging. Retina 2010; 30(9): 1441-1454.

6 Suzuki M, Curcio CA, Mullins RF, Spaide RF. Refractile drusen: clinical imaging and candidate histology. Retina 2015; 35(5): 859-865.

7 Schlanitz FG, Baumann B, Kundi M, Sacu S, Baratsits M, Scheschy U et al. Drusen volume development over time and its relevance to the course of age-related macular degeneration. Br J Ophthalmol 2016; e-pub ahead of print 4 April 2016; doi:10.1136/bjophthalmol-2016-308422.

8 Yehoshua Z, Wang F, Rosenfeld PJ, Penha FM, Feuer WJ, Gregori G. Natural history of drusen morphology in agerelated macular degeneration using spectral domain optical coherence tomography. Ophthalmology 2011; 118(12): 2434-2441

9 Querques G, Souied EH. Vascularized drusen: slowly progressive type 1 neovascularization mimicking drusenoid retinal pigment epithelium elevation. Retina 2015; 35(12): 2433-2439.

10 Querques G, Srour M, Massamba N, Georges A, Ben Moussa N, Rafaeli $\mathrm{O}$ et al. Functional characterization and multimodal imaging of treatment-naive 'quiescent' choroidal neovascularization. Invest Ophthalmol Vis Sci 2013; 54(10): 6886-6892.

11 Roisman L, Zhang Q, Wang RK, Gregori G, Zhang A, Chen CL et al. Optical coherence tomography angiography of asymptomatic neovascularization in intermediate age-related macular degeneration. Ophthalmology 2016; 123(6): 1309-1319.

12 Zweifel SA, Spaide RF, Curcio CA, Malek G, Imamura Y. Reticular pseudodrusen are subretinal drusenoid deposits. Ophthalmology 2010; 117(2): 303-12 e1.

13 Zarubina AV, Neely DC, Clark ME, Huisingh CE, Samuels BC, Zhang $\mathrm{Y}$ et al. Prevalence of subretinal drusenoid deposits in older persons with and without age-related macular degeneration, by multimodal imaging. Ophthalmology 2016; 123(5): 1090-1100.

14 Saade C, Smith RT. Reticular macular lesions: a review of the phenotypic hallmarks and their clinical significance. Clin Exp Ophthalmol 2014; 42(9): 865-874.

15 Schaal KB, Legarreta AD, Gregori G, Legarreta JE, Cheng Q, Stetson PF et al. Widefield en face optical coherence tomography imaging of subretinal drusenoid deposits. Ophthalmic Surg Lasers Imaging Retina 2015; 46(5): 550-559.

16 Schlanitz F, Baratsits M, Eibenberger K, Montuoro A, Zotter S, Baumann $B$ et al. Investigating the Development of Pseudodrusen in Age-related Macular Degeneration. ARVO Annual Meeting 3-7 May 2015; ARVO: Denver, CO, USA.
17 Querques G, Canoui-Poitrine F, Coscas F, Massamba N, Querques L, Mimoun G et al. Analysis of progression of reticular pseudodrusen by spectral domain-optical coherence tomography. Invest Ophthalmol Vis Sci 2012; 53(3): 1264-1270.

18 Christenbury JG, Folgar FA, O'Connell RV, Chiu SJ, Farsiu S, Toth CA et al. Progression of intermediate age-related macular degeneration with proliferation and inner retinal migration of hyperreflective foci. Ophthalmology 2013; 120(5): 1038-1045.

19 Folgar FA, Yuan EL, Sevilla MB, Chiu SJ, Farsiu S, Chew EY et al. Drusen volume and retinal pigment epithelium abnormal thinning volume predict 2-year progression of agerelated macular degeneration. Ophthalmology 2016; 123(1): 39-50 e1.

20 Schuman SG, Koreishi AF, Farsiu S, Jung SH, Izatt JA, Toth CA. Photoreceptor layer thinning over drusen in eyes with agerelated macular degeneration imaged in vivo with spectraldomain optical coherence tomography. Ophthalmology 2009; 116(3): 488-96 e2.

21 Ouyang Y, Heussen FM, Hariri A, Keane PA, Sadda SR. Optical coherence tomography-based observation of the natural history of drusenoid lesion in eyes with dry agerelated macular degeneration. Ophthalmology 2013; 120(12): 2656-2665.

22 Nathoo NA, Or C, Young M, Chui L, Fallah N, Kirker AW et al. Optical coherence tomography-based measurement of drusen load predicts development of advanced age-related macular degeneration. Am J Ophthalmol 2014; 158(4): 757-61 e1.

23 Wu Z, Luu CD, Ayton LN, Goh JK, Lucci LM, Hubbard WC et al. Optical coherence tomography-defined changes preceding the development of drusen-associated atrophy in age-related macular degeneration. Ophthalmology 2014; 121(12): 2415-2422.

24 Huisingh C, McGwin Jr G, Neely D, Zarubina A, Clark M, Zhang $\mathrm{Y}$ et al. The association between subretinal drusenoid deposits in older adults in normal macular health and incident age-related macular degeneration. Invest Ophthalmol Vis Sci 2016; 57(2): 739-745.

25 Spaide RF. Outer retinal atrophy after regression of subretinal drusenoid deposits as a newly recognized form of late age-related macular degeneration. Retina 2013; 33(9): $1800-1808$

26 Schaal KB, Rosenfeld PJ, Gregori G, Yehoshua Z, Feuer WJ, Anatomic Clinical Trial. Endpoints for nonexudative agerelated macular degeneration. Ophthalmology 2016; 123(5): 1060-1079.

27 Jain N, Farsiu S, Khanifar AA, Bearelly S, Smith RT, Izatt JA et al. Quantitative comparison of drusen segmented on SDOCT $v$ drusen delineated on color fundus photographs. Invest Ophthalmol Vis Sci 2010; 51(10): 4875-4883.

28 Gregori G, Wang F, Rosenfeld PJ, Yehoshua Z, Gregori NZ, Lujan BJ et al. Spectral domain optical coherence tomography imaging of drusen in nonexudative age-related macular degeneration. Ophthalmology 2011; 118(7): 1373-1379.

29 Abdelfattah NS, Zhang H, Boyer DS, Rosenfeld PJ, Feuer WJ, Gregori $\mathrm{G}$ et al. Drusen volume as a predictor of disease progression in patients with late age-related macular degeneration in the fellow eye. Invest Ophthalmol Vis Sci 2016; 57(4): 1839-1846.

30 Chiu SJ, Izatt JA, O'Connell RV, Winter KP, Toth CA, Farsiu S. Validated automatic segmentation of AMD pathology 
including drusen and geographic atrophy in SD-OCT images. Invest Ophthalmol Vis Sci 2012; 53(1): 53-61.

31 deSisternes L, Simon N, Tibshirani R, Leng T, Rubin DL. Quantitative SD-OCT imaging biomarkers as indicators of age-related macular degeneration progression. Invest Ophthalmol Vis Sci 2014; 55(11): 7093-7103.

32 Schlanitz FG, Sacu S, Baumann B, Bolz M, Platzer M, Pircher M et al. Identification of drusen characteristics in age-related macular degeneration by polarization-sensitive optical coherence tomography. Am J Ophthalmol 2015; 160(2): 335-44 e1.

33 Keane PA, Patel PJ, Liakopoulos S, Heussen FM, Sadda SR, Tufail A. Evaluation of age-related macular degeneration with optical coherence tomography. Surv Ophthalmol 2012; 57(5): 389-414.

34 Schmidt-Erfurth U, Chong V, Loewenstein A, Larsen M, Souied E, Schlingemann $\mathrm{R}$ et al. Guidelines for the management of neovascular age-related macular degeneration by the European Society of Retina Specialists (EURETINA). Br J Ophthalmol 2014; 98(9): 1144-1167.

35 Schmidt-Erfurth U, Waldstein SM. A paradigm shift in imaging biomarkers in neovascular age-related macular degeneration. Prog Retin Eye Res 2016; 50: 1-24.

36 Freund KB, Zweifel SA, Engelbert M. Do we need a new classification for choroidal neovascularization in age-related macular degeneration? Retina 2010; 30(9): 1333-1349.

37 Spaide RF. Enhanced depth imaging optical coherence tomography of retinal pigment epithelial detachment in agerelated macular degeneration. Am J Ophthalmol 2009; 147(4): 644-652.

38 Sato T, Iida T, Hagimura N, Kishi S. Correlation of optical coherence tomography with angiography in retinal pigment epithelial detachment associated with age-related macular degeneration. Retina 2004; 24(6): 910-914.

39 Kuehlewein L, Bansal M, Lenis TL, Iafe NA, Sadda SR, Bonini Filho MA et al. Optical coherence tomography angiography of type 1 neovascularization in age-related macular degeneration. Am J Ophthalmol 2015; 160(4): $739-48$ e2.

40 Schmidt-Erfurth U, Waldstein SM, Deak GG, Kundi M, Simader C. Pigment epithelial detachment followed by retinal cystoid degeneration leads to vision loss in treatment of neovascular age-related macular degeneration. Ophthalmology 2015; 122(4): 822-832.

41 Simader C, Ritter M, Bolz M, Deak GG, Mayr-Sponer U, Golbaz I et al. Morphologic parameters relevant for visual outcome during anti-angiogenic therapy of neovascular agerelated macular degeneration. Ophthalmology 2014; 121(6): 1237-1245.

42 Dirani A, Ambresin A, Marchionno L, Decugis D, Mantel I. Factors influencing the treatment response of pigment epithelium detachment in age-related macular degeneration. Am J Ophthalmol 2015; 160(4): 732-8 e2.

43 Querques G, Atmani K, Berboucha E, Martinelli D, Coscas G, Soubrane $\mathrm{G}$ et al. Angiographic analysis of retinal-choroidal anastomosis by confocal scanning laser ophthalmoscopy technology and corresponding (eye-tracked) spectraldomain optical coherence tomography. Retina 2010; 30(2): 222-234.

44 Nagiel A, Sarraf D, Sadda SR, Spaide RF, Jung J, Bhavsar KV et al. Type 3 neovascularization: evolution, association with pigment epithelial detachment, and treatment response as revealed by spectral domain optical coherence tomography. Retina 2015; 35(4): 638-647.
45 Grunwald JE, Daniel E, Huang J, Ying GS, Maguire MG, Toth CA et al. Risk of geographic atrophy in the comparison of age-related macular degeneration treatments trials. Ophthalmology 2014; 121(1): 150-161.

46 Kuehlewein L, Dansingani KK, de Carlo TE, Bonini Filho MA, Iafe NA, Lenis TL et al. Optical coherence tomography angiography of type 3 neovascularization secondary to agerelated macular degeneration. Retina 2015; 35(11): 2229-2235.

47 Roberts P, Sugita M, Deak G, Baumann B, Zotter S, Pircher M et al. Automated identification and quantification of subretinal fibrosis in neovascular age-related macular degeneration using polarization-sensitive OCT. Invest Ophthalmol Vis Sci 2016; 57(4): 1699-1705.

48 Shah VP, Shah SA, Mrejen S, Freund KB. Subretinal hyperreflective exudation associated with neovascular agerelated macular degeneration. Retina 2014; 34(7): 1281-1288.

49 Willoughby AS, Ying GS, Toth CA, Maguire MG, Burns RE, Grunwald JE et al. Subretinal hyperreflective material in the comparison of age-related macular degeneration treatments trials. Ophthalmology 2015; 122(9): 1846-53 e5.

50 Dansingani KK, Tan A, Gilani F, Phasukkijwatana N, Novais E, Querques L et al. Subretinal hyperreflective material imaged with optical coherence tomography angiography. Am J Ophthalmol 2016; 169: 235-248.

51 Abri Aghdam K, Pielen A, Framme C, Junker B. Correlation between hyperreflective foci and clinical outcomes in neovascular age-related macular degeneration after switching to aflibercept. Invest Ophthalmol Vis Sci 2015; 56(11): 6448-6455.

52 Akagi-Kurashige Y, Tsujikawa A, Oishi A, Ooto S, Yamashiro K, Tamura $\mathrm{H}$ et al. Relationship between retinal morphological findings and visual function in age-related macular degeneration. Graefes Arch Clin Exp Ophthalmol 2012; 250(8): 1129-1136.

53 Lee H, Ji B, Chung H, Kim HC. Correlation between optical coherence tomographic hyperreflective foci and visual outcomes after anti-Vegf treatment in neovascular agerelated macular degeneration and polypoidal choroidal vasculopathy. Retina 2016; 36(3): 465-475.

54 Segal O, Barayev E, Nemet AY, Geffen N, Vainer I, Mimouni M. Prognostic value of hyperreflective foci in neovascular agerelated macular degeneration treated with bevacizumab. Retina 2016; 36(11): 2175-2182.

55 Ritter M, Simader C, Bolz M, Deak GG, Mayr-Sponer U, Sayegh $\mathrm{R}$ et al. Intraretinal cysts are the most relevant prognostic biomarker in neovascular age-related macular degeneration independent of the therapeutic strategy. $\mathrm{Br} \mathrm{J}$ Ophthalmol 2014; 98(12): 1629-1635.

56 Jaffe GJ, Martin DF, Toth CA, Daniel E, Maguire MG, Ying GS et al. Macular morphology and visual acuity in the comparison of age-related macular degeneration treatments trials. Ophthalmology 2013; 120(9): 1860-1870.

57 Sharma S, Toth CA, Daniel E, Grunwald JE, Maguire MG, Ying GS et al. Macular morphology and visual acuity in the second year of the comparison of age-related macular degeneration treatments trials. Ophthalmology 2016; 123(4): 865-875.

58 Waldstein SM, Philip AM, Leitner R, Simader C, Langs G, Gerendas BS et al. Correlation of 3-dimensionally quantified intraretinal and subretinal fluid with visual acuity in neovascular age-related macular degeneration. JAMA Ophthalmol 2016; 134(2): 182-190.

59 Waldstein SM, Wright J, Warburton J, Margaron P, Simader C, Schmidt-Erfurth U. Predictive value of retinal morphology for 
visual acuity outcomes of different ranibizumab treatment regimens for neovascular AMD. Ophthalmology 2016; 123(1): 60-69.

60 Gianniou C, Dirani A, Jang L, Mantel I. Refractory intraretinal or subretinal fluid in neovascular age-related macular degeneration treated with intavitreal ranibizumab: functional and structural outcome. Retina 2015; 35(6): 1195-1201.

61 Penha FM, Gregori G, Garcia Filho CA, Yehoshua Z, Feuer WJ, Rosenfeld PJ. Quantitative changes in retinal pigment epithelial detachments as a predictor for retreatment with anti-VEGF therapy. Retina 2013; 33(3): 459-466.

62 Or C, Chui L, Fallah N, Forooghian F. Volumetric assessment of the responsiveness of pigment epithelial detachments in neovascular age-related macular degeneration to intravitreal bevacizumab. Retina 2016; 36(2): 264-271.

63 Nagiel A, Freund KB, Spaide RF, Munch IC, Larsen M, Sarraf D. Mechanism of retinal pigment epithelium tear formation following intravitreal anti-vascular endothelial growth factor therapy revealed by spectral-domain optical coherence tomography. Am J Ophthalmol 2013; 156(5): 981-8 e2.

64 Sarraf D, Chan C, Rahimy E, Abraham P. Prospective evaluation of the incidence and risk factors for the development of RPE tears after high- and low-dose ranibizumab therapy. Retina 2013; 33(8): 1551-1557.

65 Waldstein SM, Simader C, Staurenghi G, Chong NV, Mitchell P, Jaffe GJ et al. Morphology and visual acuity in aflibercept and ranibizumab therapy for neovascular agerelated macular degeneration in the VIEW trials. Ophthalmology 2016; 123(7): 1521-1529.

66 Sulzbacher F, Roberts P, Munk MR, Kaider A, Kroh ME, Sacu $S$ et al. Relationship of retinal morphology and retinal sensitivity in the treatment of neovascular age-related macular degeneration using aflibercept. Invest Ophthalmol Vis Sci 2015; 56(2): 1158-1167.

67 Mayr-Sponer U, Waldstein SM, Kundi M, Ritter M, Golbaz I, Heiling $\mathrm{U}$ et al. Influence of the vitreomacular interface on outcomes of ranibizumab therapy in neovascular age-related macular degeneration. Ophthalmology 2013; 120(12): 2620-2629.

68 Waldstein SM, Ritter M, Simader C, Mayr-Sponer U, Kundi M, Schmidt-Erfurth U. Impact of vitreomacular adhesion on ranibizumab mono- and combination therapy for neovascular age-related macular degeneration. Am J Ophthalmol 2014; 158(2): 328-36 e1.

69 Takahashi H, Nomura Y, Tan X, Fujino Y, Kawashima H, Yanagi $Y$. Effects of posterior vitreous detachment on aqueous humour levels of VEGF and inflammatory cytokines. Br J Ophthalmol 2015; 99(8): 1065-1069.

70 Coscas G, De Benedetto U, Coscas F, Li Calzi CI, Vismara S, Roudot-Thoraval F et al. Hyperreflective dots: a new spectral-domain optical coherence tomography entity for follow-up and prognosis in exudative age-related macular degeneration. Ophthalmologica 2013; 229(1): 32-37.

71 Channa R, Sophie R, Bagheri S, Shah SM, Wang J, Adeyemo O et al. Regression of choroidal neovascularization results in macular atrophy in anti-vascular endothelial growth factortreated eyes. Am J Ophthalmol 2015; 159(1): 9-19 e1-2.

$72 \mathrm{Xu} \mathrm{L}$, Mrejen S, Jung JJ, Gallego-Pinazo R, Thompson D, Marsiglia $\mathrm{M}$ et al. Geographic atrophy in patients receiving anti-vascular endothelial growth factor for neovascular agerelated macular degeneration. Retina 2015; 35(2): 176-186.
73 Abdelfattah NS, Zhang H, Boyer DS, Sadda SR. Progression of macular atrophy in patients with neovascular age-related macular degeneration undergoing antivascular endothelial growth factor therapy. Retina 2016.

74 Arnold JJ, Markey CM, Kurstjens NP, Guymer RH. The role of sub-retinal fluid in determining treatment outcomes in patients with neovascular age-related macular degeneration-a phase IV randomised clinical trial with ranibizumab: the FLUID study. BMC Ophthalmol 2016; 16: 31.

75 Shi F, Chen X, Zhao H, Zhu W, Xiang D, Gao E et al. Automated 3-D retinal layer segmentation of macular optical coherence tomography images with serous pigment epithelial detachments. IEEE Trans Med Imaging 2015; 34(2): 441-452.

76 Zhang L, Sonka M, Folk JC, Russell SR, Abramoff MD. Quantifying disrupted outer retinal-subretinal layer in $\mathrm{SD}-\mathrm{OCT}$ images in choroidal neovascularization. Invest Ophthalmol Vis Sci 2014; 55(4): 2329-2335.

77 Xu X, Lee K, Zhang L, Sonka M, Abramoff M. Stratified sampling voxel classification for segmentation of intraretinal and subretinal fluid in longitudinal clinical OCT data. IEEE Trans Med Imaging 2015; 34(7): 1616-1623.

78 Schlegl T, Waldstein SM, Vogl WD, Schmidt-Erfurth U, Langs G. Predicting semantic descriptions from medical images with convolutional neural networks. Inf Process Med Imaging 2015; 24: 437-448.

79 Bogunovic H, Abramoff M, Zhang L, Sonka M. Prediction of Treatment Response from Retinal OCT in Patients with Exudative Age-Related Macular Degeneration. International Workshop on Ophthalmic Medical Image Analysis of MICCAI: Boston, MA, USA, 2014.

80 Vogl WD, Waldstein SM, Gerendas BS, Simader C, Glodan AM, Podkowinski D et al. Spatio-temporal signatures to predict retinal disease recurrence. Inf Process Med Imaging 2015; 24: 152-163.

81 Fleckenstein M, Charbel Issa P, Helb HM, SchmitzValckenberg S, Finger RP, Scholl HP et al. High-resolution spectral domain-OCT imaging in geographic atrophy associated with age-related macular degeneration. Invest Ophthalmol Vis Sci 2008; 49(9): 4137-4144.

82 Yehoshua Z, Rosenfeld PJ, Gregori G, Feuer WJ, Falcao M, Lujan BJ et al. Progression of geographic atrophy in agerelated macular degeneration imaged with spectral domain optical coherence tomography. Ophthalmology 2011; 118(4): 679-686.

83 Holz FG, Bindewald-Wittich A, Fleckenstein M, Dreyhaupt J, Scholl HP, Schmitz-Valckenberg S. Progression of geographic atrophy and impact of fundus autofluorescence patterns in age-related macular degeneration. $A m \mathrm{~J}$ Ophthalmol 2007; 143(3): 463-472.

84 Fleckenstein M, Schmitz-Valckenberg S, Adrion C, Kramer I, Eter N, Helb HM et al. Tracking progression with spectraldomain optical coherence tomography in geographic atrophy caused by age-related macular degeneration. Invest Ophthalmol Vis Sci 2010; 51(8): 3846-3852.

85 Simader C, Sayegh RG, Montuoro A, Azhary M, Koth AL, Baratsits $\mathrm{M}$ et al. A longitudinal comparison of spectraldomain optical coherence tomography and fundus autofluorescence in geographic atrophy. Am J Ophthalmol 2014; 158(3): 557-66 e1.

86 Schmitz-Valckenberg S, Fleckenstein M, Helb HM, Charbel Issa P, Scholl HP, Holz FG. In vivo imaging of foveal sparing in geographic atrophy secondary to age-related macular 
degeneration. Invest Ophthalmol Vis Sci 2009; 50(8): 3915-3921.

87 Bearelly S, Chau FY, Koreishi A, Stinnett SS, Izatt JA, Toth CA. Spectral domain optical coherence tomography imaging of geographic atrophy margins. Ophthalmology 2009; 116(9): 1762-1769.

88 Stetson PF, Yehoshua Z, Garcia Filho CA, Portella Nunes R, Gregori G, Rosenfeld PJ. OCT minimum intensity as a predictor of geographic atrophy enlargement. Invest Ophthalmol Vis Sci 2014; 55(2): 792-800.

89 Moussa K, Lee JY, Stinnett SS, Jaffe GJ. Spectral domain optical coherence tomography-determined morphologic predictors of age-related macular degeneration-associated geographic atrophy progression. Retina 2013; 33(8): 1590-1599.

90 Hariri AH, Nittala MG, Sadda SR. Quantitative characteristics of spectral-domain optical coherence tomography in corresponding areas of increased autofluorescence at the margin of geographic atrophy in patients with age-related macular degeneration. Ophthalmic Surg Lasers Imaging Retina 2016; 47(6): 523-527.

91 Fleckenstein M, Schmitz-Valckenberg S, Martens C, Kosanetzky S, Brinkmann CK, Hageman GS et al. Fundus autofluorescence and spectral-domain optical coherence tomography characteristics in a rapidly progressing form of geographic atrophy. Invest Ophthalmol Vis Sci 2011; 52(6): 3761-3766.

92 Choi W, Moult EM, Waheed NK, Adhi M, Lee B, Lu CD et al. Ultrahigh-speed, swept-source optical coherence tomography angiography in nonexudative age-related macular degeneration with geographic atrophy.

Ophthalmology 2015; 122(12): 2532-2544.

93 Schutze C, Bolz M, Sayegh R, Baumann B, Pircher M, Gotzinger $\mathrm{E}$ et al. Lesion size detection in geographic atrophy by polarization-sensitive optical coherence tomography and correlation to conventional imaging techniques. Invest Ophthalmol Vis Sci 2013; 54(1): 739-745.

94 Sayegh RG, Zotter S, Roberts PK, Kandula MM, Sacu S, Kreil DP et al. Polarization-sensitive optical coherence tomography and conventional retinal imaging strategies in assessing foveal integrity in geographic atrophy. Invest Ophthalmol Vis Sci 2015; 56(9): 5246-5255.

95 Niu S, de Sisternes L, Chen Q, Leng T, Rubin DL. Automated geographic atrophy segmentation for SD-OCT images using region-based C-V model via local similarity factor. Biomed Opt Express 2016; 7(2): 581-600.

96 Chen Q, de Sisternes L, Leng T, Zheng L, Kutzscher L, Rubin DL. Semi-automatic geographic atrophy segmentation for SD-OCT images. Biomed Opt Express 2013; 4(12): 2729-2750.

97 Hu Z, Medioni GG, Hernandez M, Hariri A, Wu X, Sadda SR. Segmentation of the geographic atrophy in spectral-domain optical coherence tomography and fundus autofluorescence images. Invest Ophthalmol Vis Sci 2013; 54(13): 8375-8383.

98 Niu S, de Sisternes L, Chen Q, Rubin DL, Leng T. Fully automated prediction of geographic atrophy growth using quantitative spectral-domain optical coherence tomography biomarkers. Ophthalmology 2016; 123(8): 1737-1750. 